\title{
PLANETARY NEBULA KINEMATICS IN M31
}

\author{
Denise Hurley-Keller, ${ }^{1,2}$ Heather L. Morrison, ${ }^{3}$ and Paul Harding ${ }^{1}$ \\ Astronomy Department, Case Western Reserve University, 10900 Euclid Avenue, Cleveland, OH 44106; \\ denise@smaug.astr.cwru.edu, heather@vegemite.astr.cwru.edu, harding@dropbear.astr.cwru.edu
}

AND

George H. JACOBY

WIYN Observatory, P.O. Box 26732, Tucson, AZ 85726; jacoby@wiyn.org Received 2003 October 1; accepted 2004 August 4

\begin{abstract}
We present kinematics of 135 planetary nebulae (PNs) in M31 from a survey covering $3.9 \mathrm{deg}^{2}$ and extending out to $15 \mathrm{kpc}$ from the southwest major axis and more than $20 \mathrm{kpc}$ along the minor axis. The majority of our sample, even well outside the disk, shows significant rotational support (mean line-of-sight velocity $116 \mathrm{~km} \mathrm{~s}^{-1}$ ). We argue that these PNs belong to the outer part of M31's large $R^{1 / 4}$ bulge. Only five PNs have velocities clearly inconsistent with this fast rotating bulge. All five may belong to tidal streams in M31's outer halo. One is projected on the Northern Spur and is counterrotating with respect to the disk there. Two are projected near the major axis at $X=-10 \mathrm{kpc}$ and have M32-like velocities; they could be debris from that galaxy. The remaining two halo PNs are located near the center of the galaxy, and their velocities follow the gradient found by Ibata and coworkers, implying that these PNs could belong to the Southern Stream. If M31 has a nonrotating, pressure-supported halo, we have yet to find it, and it must be a very minor component of the galaxy.
\end{abstract}

Subject headings: galaxies: halos — galaxies: individual (M31) — galaxies: kinematics and dynamics galaxies: stellar content

On-line material: color figures

\section{INTRODUCTION}

The wealth of information resulting from highly detailed studies of the old stellar populations of the Galaxy (Eggen et al. 1962; Searle \& Zinn 1978; Gilmore \& Reid 1983; Edvardsson et al. 1993) has been a catalyst for theories of galaxy evolution; as the nearest large galaxy to us, M31 is a proving ground for those theories. M31 offers a variety of stellar populations in a galaxy of earlier Hubble type, providing important leverage in testing formation scenarios. Especially important are the faint, old populations of the halo, which have proven difficult to study directly in more distant galaxies (Morrison 1999; Zepf et al. 2000). Halos contain the first stars, and the ages and abundances of these stars reflect the properties of the protogalactic fragments that are the building blocks of galaxies (e.g., Oey 2000). The kinematics of halo populations tells us about the dynamical evolution of the galaxy and provides constraints on hierarchical formation theories (Helmi et al. 1999; Ivezić et al. 2000; Yanny et al. 2000; Dohm-Palmer et al. 2001).

How can we describe M31's old populations? What can their properties tell us about how M31 formed and evolved? The old stellar populations in the Milky Way have often been used as a guide to these populations in other disk galaxies because of the richness of spatial and velocity data for the Milky Way. In the Milky Way, the metal-poor, old stars of the halo dominate a few kiloparsecs away from the plane and the Galactic center. The field stars and globular clusters have a power-law density

\footnotetext{
1 Visiting Astronomer, Kitt Peak National Observatory, National Optical Astronomy Observatory, which is operated by the Association of Universities for Research in Astronomy, Inc. (AURA), under cooperative agreement with the National Science Foundation.

2 NSF Astronomy and Astrophysics Postdoctoral Fellow.

3 Cottrell Scholar of Research Corporation and NSF CAREER fellow.
}

profile (Zinn 1985; Saha 1985; Ivezić et al. 2000) and pressuresupported kinematics with little or no rotation (also see Beers et al. 2000 for a recent summary of halo kinematics in the Milky Way).

This density profile differs from that of M31's spheroid, which has an $R^{1 / 4}$ profile extending unchanged for a remarkably large distance along the minor axis $(20 \mathrm{kpc}$, Pritchet $\&$ van den Bergh 1994, hereafter PvdB94; 30 kpc, Durrell et al. 2004). Color-magnitude diagram studies of red giants have shown that, although there is a weak $(10 \%-20 \%)$ metal-poor component, the dominant stellar population at these halo-like distances is about 1 dex more metal-rich than its Milky Way counterpart or than the M31 globular clusters at the same radius (Mould \& Kristian 1986; Durrell et al. 1994, 2001; Couture et al. 1995; Rich et al. 1996). This led Mould (1986) and Freeman (1996) to suggest that the bulge dominates even at these very large distances from the galactic center.

The Milky Way's bulge is quite different. It is less luminous, and its inner $1-2 \mathrm{kpc}$ are dominated by a bar, which has a vertical exponential distribution with a scale height of around 350 pc (Weiland et al. 1994). Small bulges like that of the Milky Way may have formed by secular processes involving central bars (Pfenniger \& Norman 1990; Courteau et al. 1996), tying the evolution of the bulge to the disk. M31's bulge is too large to have formed by this mechanism.

The question of the difference between a large $R^{1 / 4}$ bulge with moderate rotational support and a nonrotating halo is not just one of taxonomy: the formation processes of these two populations are quite different. A kinematically hot, nonrotating stellar halo is most simply formed after the aggregation of the galaxy's dark halo - this explains the lack of correlation between the angular momenta of the halo and the disk (Freeman 1996). This scenario is supported by growing evidence that the 
Milky Way halo was populated, at least in part, by the accretion and destruction of small satellites (Majewski et al. 1994; Helmi et al. 1999; Ivezić et al. 2000; Yanny et al. 2000; Dohm-Palmer et al. 2001). The Sgr dwarf (Ibata et al. 1994) and its tidal streams give a present-day example of this process.

However, the accretion history of the Milky Way may be dramatically different from that of a galaxy of earlier Hubble type such as M31. An $R^{1 / 4}$ profile is a result of violent relaxation such as occurs, for example, in major mergers. We note that both accretion and merging involve interaction between galaxies, but the mass ratios are very different: in the merger that forms an $R^{1 / 4}$ law profile, the mass ratio is at most a few to 1 , while the mass ratio for the accretion of small satellites to form a hot halo is more typically 100:1 or more. As a result, major mergers have a strong effect on the galaxy disk while the accretions have almost none.

Does M31 actually have a kinematically hot halo? If so, how much luminosity does it contribute? Accurate kinematic data for halo objects are needed to quantify the relative importance of this process in populating the outer regions of M31. However, the difficulty in isolating the halo from the bulge and in obtaining accurate velocities for halo populations has precluded a definitive answer to these questions. Spectroscopy of single field stars in M31 is very challenging, even for giant branch tip stars using Keck, since samples are dominated by contamination by foreground stars from the Milky Way (Reitzel et al. 1998; Reitzel \& Guhathakurta 2002).

Globular clusters have been the halo tracer of choice thus far, but they present several difficulties. Cluster velocities derive from absorption spectra, and accurate velocities (errors $<20 \mathrm{~km} \mathrm{~s}^{-1}$ ) are only now becoming available (Perrett et al. 2002; P. Seitzer 2002, private communication). Thus, early studies of M31 cluster kinematics concluded that its halo had little rotation (Huchra et al. 1991; Huchra 1993), while more recent studies find greater rotational support (Perrett et al. 2002). In the Milky Way, the field stars and globular clusters share a similar metallicity distribution (Zinn 1985; Laird et al. 1988; Ryan \& Norris 1991) and "hot" kinematics (Norris 1986; Beers et al. 2000). However, in M31 the abundance distributions of the clusters and the field stars at similar radii are quite different; the clusters are more metal-poor in the mean than the field stars (Durrell et al. 2001; Barmby et al. 2000). Because cluster metallicities differ, cluster kinematics also may not be entirely representative of the stellar halo properties.

Planetary nebulae (PNs), in contrast, trace stellar populations with ages $\sim 1-10$ Gyr and readily give accurate velocities with $4 \mathrm{~m}$ class telescopes because almost all their energy output is concentrated in a few emission lines. The techniques for detecting PNs have been well documented by G. Jacoby and collaborators (Jacoby et al. 1989). PNs have been used extensively as kinematic tracers in normal elliptical galaxies (Hui et al. 1995; Arnaboldi et al. 1996; Méndez et al. 2001) and in the luminous members of the Local Group (Nolthenius \& Ford 1986, 1987). Nolthenius \& Ford (1987, hereafter NF87) presented kinematics of 37 M31 PNs, with distances along the major axis of up to $40 \mathrm{kpc}$ and distances from the major axis of up to $17 \mathrm{kpc}$. Although it is possible to identify the signatures of disk and bulge in their data and find a few objects with clear halo kinematics, the small size of their sample precludes strong conclusions.

Here we present the results of a kinematic survey of 135 PNs in one quadrant of M31's outer bulge/halo. The survey extends to $20 \mathrm{kpc}$ along the minor axis, well into the canonical halo regions of $\mathrm{M} 31$, and $20 \mathrm{kpc}$ along the southwest major axis. We have used both the spatial and kinematic information for the PNs to distinguish the bulge population from the halo population. With this larger number of PNs and the accurate velocities (errors of 5-10 $\mathrm{km} \mathrm{s}^{-1}$ ) resulting from the followup Hydra spectroscopy, we have a clearer picture of M31's spheroid. Using the Milky Way as a guide, we expected to find only a small degree of rotational support in the populations far from the major axis, similar to the Milky Way's stellar halo. Instead, we find that the bulge dominates out to the limits of our survey and that very few objects belong to a nonrotating, pressure-supported halo like that of the Milky Way.

In $\S 2$ we describe the survey that identified our PNs and the spectroscopic follow-up to obtain velocities. In $\S 3$ we present the PN kinematics and discuss the spatial and kinematic signatures of the thin disk, halo, and bulge in M31 in relation to these results, concluding that a thin disk and a dominant bulge best explain our data. In $\S 4$, we interpret the kinematics in the context of formation scenarios for these populations.

\section{OBSERVATIONS}

\subsection{PN Detection and Photometry}

Our PN detection relies on the fact that PNs have a very strong $\mathrm{O}$ III emission line at $5007 \AA$ and relatively little continuum emission. Distant PNs can therefore be detected via narrowband imaging at $5007 \AA$. Exposures at a nearby but offset wavelength can be used to discriminate PNs from stars.

Imaging of M31 was carried out at the Case Western Reserve University Burrell Schmidt on the nights of 2000 September 23-30. The camera at the Schmidt has a SITe $2 \mathrm{k} \times 4 \mathrm{k}$ backilluminated CCD with $15 \mu \mathrm{m}$ pixels and a pixel scale of $\sim 1^{\prime \prime} 45$ pixel $^{-1}$. It covers a $40^{\prime}(\mathrm{E}-\mathrm{W}) \times 80^{\prime}(\mathrm{N}-\mathrm{S})$ field. The read noise during the Schmidt imaging run was $12 e^{-}$, and the gain was $1.8 e^{-} \mathrm{ADU}^{-1}$. The seeing $\left(2^{\prime \prime}-2\right.$ ".5) was typical for the Schmidt.

The choice of filter depends on several factors, and some issues regarding filters are discussed in Jacoby et al. (1992). The [O III] $\lambda 5007$ filter should be narrow enough to minimize the sky contribution yet wide enough to accommodate the potentially large range of velocities. It should also be well matched to the observed wavelength of the $[\mathrm{O}$ III $] \lambda 5007$ emission line. M31's systemic velocity is $-300 \mathrm{~km} \mathrm{~s}^{-1}$ (de Vaucouleurs et al. 1991), so the [O III] 25007 line is shifted blueward to $5002 \AA$. A blueward shift of $13 \AA$ in the filter response results from the Burrell Schmidt's $\mathrm{f} / 3.5$ beam (this accounts for a shift of $\sim 10 \AA$ ) and the temperature difference between the lab where the filter response curve published by NOAO was measured and the observing conditions $(\sim 3 \AA)$. For fields 1 and 2 , we used filter KP1590 with $\lambda_{c}=5022 \AA$, and for field 3, filter KP1467 with $\lambda_{c}=5008 \AA$.

The filter transmission for a velocity of $\pm 400 \mathrm{~km} \mathrm{~s}^{-1}$ with respect to M31's systemic velocity (the likely worst case) is $\gtrsim 70 \%$ in all cases (Fig. 1). We note that the filter used for field 3 has a $15 \%$ gradient across the velocities of interest. Detection of disk PNs is unaffected, since the field is located on the approaching side of the galaxy and the emission is blueshifted into the higher sensitivity region of the transmission curve. In addition, the gradient across disk velocities will be negligible because of the relatively low dispersion of disk PNs $\left(<100 \mathrm{~km} \mathrm{~s}^{-1}\right)$. Detection of halo objects may have been slightly biased against those with velocities more than $200 \mathrm{~km}$ $\mathrm{s}^{-1}$ greater than the systemic velocity in that field. Although 


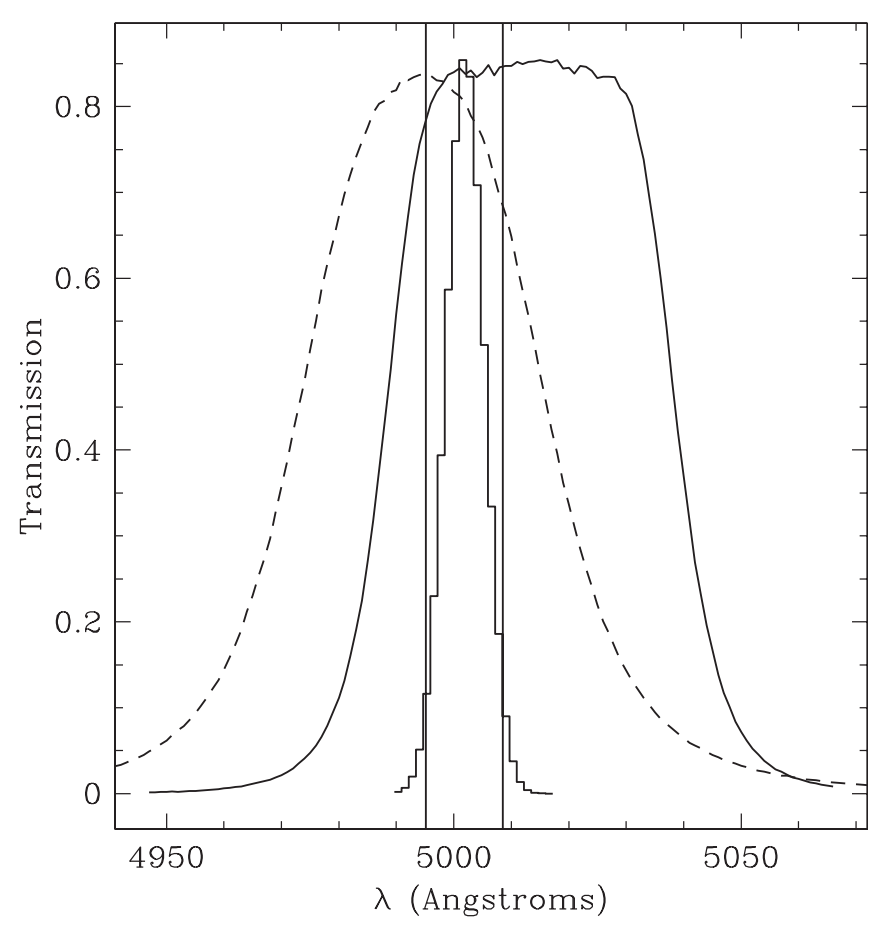

FIG. 1.-Filter transmission curves for this survey. Solid line: Curve for KP1590, used for fields 1 and 2. Dashed line: Curve for KP1467, used for field 3. Both curves have been shifted $13 \AA$ blueward to account for the $f / 3.5$ beam of the Schmidt and the observing temperature. The histogram is a Gaussian distribution with mean -300 and $\sigma=200 \mathrm{~km} \mathrm{~s}^{-1}$ representing the expected M31 halo velocity distribution. The vertical lines show the location of velocities $\pm 400 \mathrm{~km} \mathrm{~s}^{-1}$ from M31's systemic velocity.

we have not quantified this effect, it would not alter our final conclusions.

We placed our fields to sample halo PNs and to measure their rotation. Fields 1 and 2 lie along the minor axis of M31, and field 3 covers a region at larger radius that includes the major axis. Figure 2 shows their placement on an image of M31 kindly provided by R. Walterbos (2003, private communication). Field 1 was observed under photometric conditions; fields 2 and 3 were observed during periods of thin cirrus and occasional heavy cloud. Fortunately, these observations are still usable because the focus of this project is the PN velocities rather than the photometry. We followed standard image reduction procedures. The images were biassubtracted and flat-fielded and then registered using bright stars in the field. We averaged them using $\mathrm{IRAF}^{4}$ imcombine with ccdclip rejection.

Because we were primarily interested in the kinematics of the halo and populations, we wanted to exclude $\mathrm{H}$ II regions from our sample as much as possible, as they belong to the young, thin disk. The geometry discussed in $\S 3.2 .1$ shows that $\mathrm{H}$ II regions confined to the disk should be rare more than about $3 \mathrm{kpc}$ from the major axis. Therefore, we limited our selection of PNs from the Schmidt data to those more than $3 \mathrm{kpc}$ from the major axis near the center of the galaxy. At larger radius, where the background light from the disk was fainter, it was easier to distinguish pointlike PNs from more extended H II regions, and we allowed the sample to extend to the major axis.

\footnotetext{
4 IRAF is distributed by the National Optical Astronomy Observatory, which is operated by the Association of Universities for Research in Astronomy, Inc. (AURA), under cooperative agreement with the National Science Foundation.
}

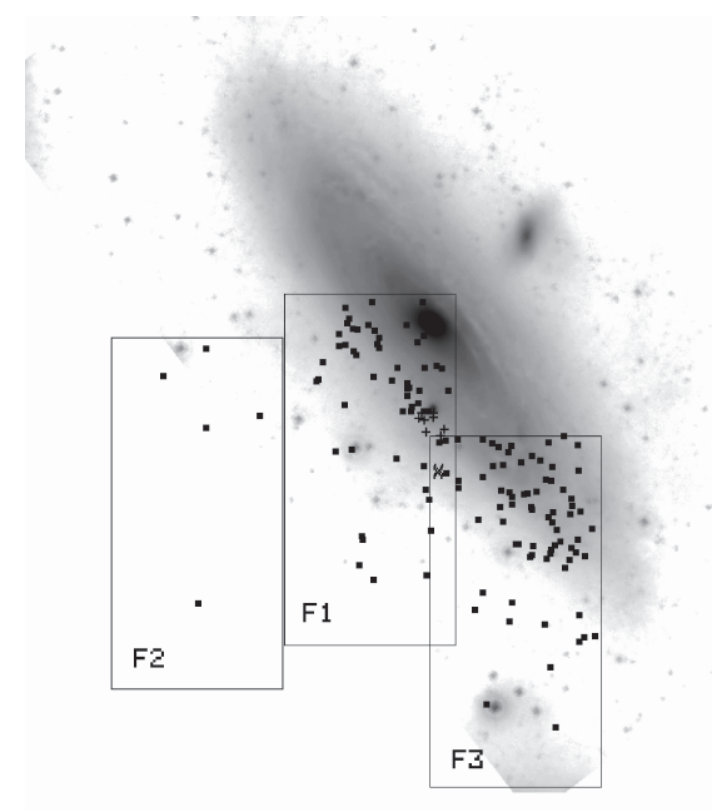

FIG. 2.-Positions of our Schmidt fields and PNs in M31. The $R$-band image was kindly provided by R. Walterbos. North is up, and east is to the left. M31's position angle is $37^{\circ} .3$ (de Vaucouleurs 1958). The southwest end of the galaxy is approaching, and the far side is to the southeast of the major axis. Squares: PNs in M31. Plus signs: PNs in M32. Crosses: H II regions in And IV.

Jacoby, Ford, \& Hui kindly shared positions for some of the objects from their earlier survey of the M31 disk and bulge (Hui et al. 1994). During the follow-up spectroscopy run, we assigned spare fibers to these objects, some of which are nearer to the center than the Schmidt sample.

To identify PN candidates, we generally followed the procedure outlined by other PN studies such as Ciardullo et al. (1989). For our project, however, it was not possible to perform photometry on a difference of the on-band image and the appropriately scaled off-band image. This is because the pointspread function (PSF) varies across the field of view of the Schmidt-because of the curved focal plane-and depends sensitively on the seeing and focus. Large residuals on the difference image would lead to many false detections. Instead, we used DoPHOT (Schechter et al. 1993) on the combined on- and off-band images separately and matched the resulting object lists. We then matched the positions of objects in the on-band image with objects in the off-band image within a 2 pixel radius. Objects in the on-band image that had no counterpart in the off-band object list were classified as PN candidates.

All candidates were visually verified. To be included in our list of spectroscopic targets, a point source in the on-band image should have no discernible flux at the same position in the offband image. Faint candidates were also required to have at least some signal present in each of the four exposures taken under the most transparent conditions. We ranked the PN candidates by the degree to which they satisfied all the criteria. As we discuss below, $\sim 88 \%$ of our first-priority PN candidates were confirmed spectroscopically, validating our selection criteria. Figure 3 shows the PN luminosity functions for confirmed PNs in fields 1 and 3 . The rough calibration is based only on assigning the brightest PNs to $M_{5007}=-4.5$ (Ciardullo et al. 1989). We reached basically to equal depths of 1.5-2 mag down the PN luminosity function in these two fields. There were only five objects in field 2, and their photometry is consistent with the other two fields. 


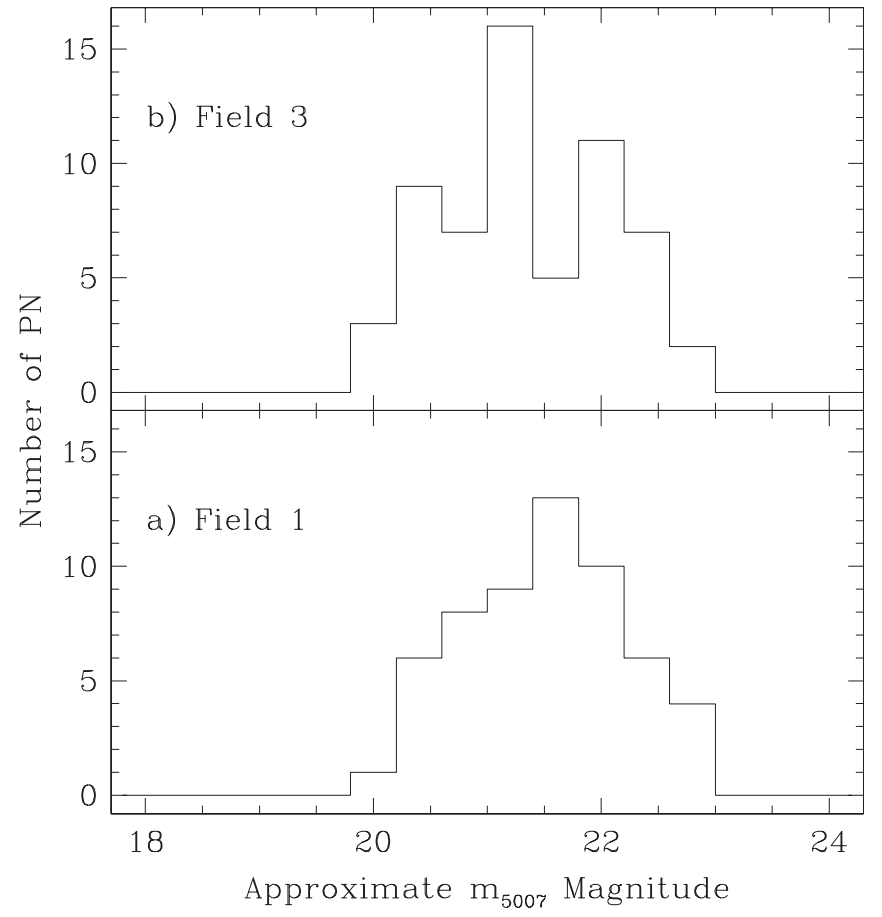

FIG. 3.-Estimated PN luminosity function for (a) field 1 and $(b)$ field 3 . The $X$-axis is the instrumental [O III] $\lambda 5007$ distribution shifted so that the bright edge of the distribution is at $m_{5007}=-4.5$.

\subsection{Astrometry}

The WIYN ${ }^{5}$ Hydra fiber spectroscopy of these candidates requires positions accurate to better than $1^{\prime \prime}$. We identified astrometric standards from the USNO-A 2.0 astrometric cata$\log$ (Monet et al. 1998) in each of our three fields. From these reference stars, we computed the transformation between the DoPHOT coordinates and the celestial coordinate system using the tfinder and ccmap packages in IRAF. With $\gtrsim 1000$ reference stars in each field and the high accuracy of the catalog positions, an accurate transformation was possible even over the large field of the Schmidt. The rms error in the calculated positions was less than 0.3 in all fields.

\subsection{Spectroscopy}

The spectroscopic observations were obtained with the WIYN Hydra fiber spectrograph on the night of 2001 September 7. The conditions were clear with good seeing, and we were able to obtain spectra for all of our candidates. For the instrument configuration, we used the red fiber set with the 600 line $\mathrm{mm}^{-1}$ grating in first order. This provided a wavelength range from 4800 to $6800 \AA$, a dispersion of $1.4 \AA$ pixel $^{-1}$, and a resolution of $2.9 \AA$.

The three Schmidt fields were divided into six separate Hydra fiber setups. For each setup, we took a series of four $600 \mathrm{~s}$ exposures and a copper-argon $(\mathrm{Cu}-\mathrm{Ar})$ lamp exposure. At the beginning of the night, we took a series of dome lamp flatfield exposures at a neutral position. More accurate flat-fielding was unnecessary, as the PNs in our sample are relatively bright and we are only interested in velocities. The spectra were overscan subtracted, trimmed, and bias subtracted via standard

\footnotetext{
5 The WIYN Observatory is a joint facility of the University of WisconsinMadison, Indiana University, Yale University, and the National Optical Astronomy Observatory.
}

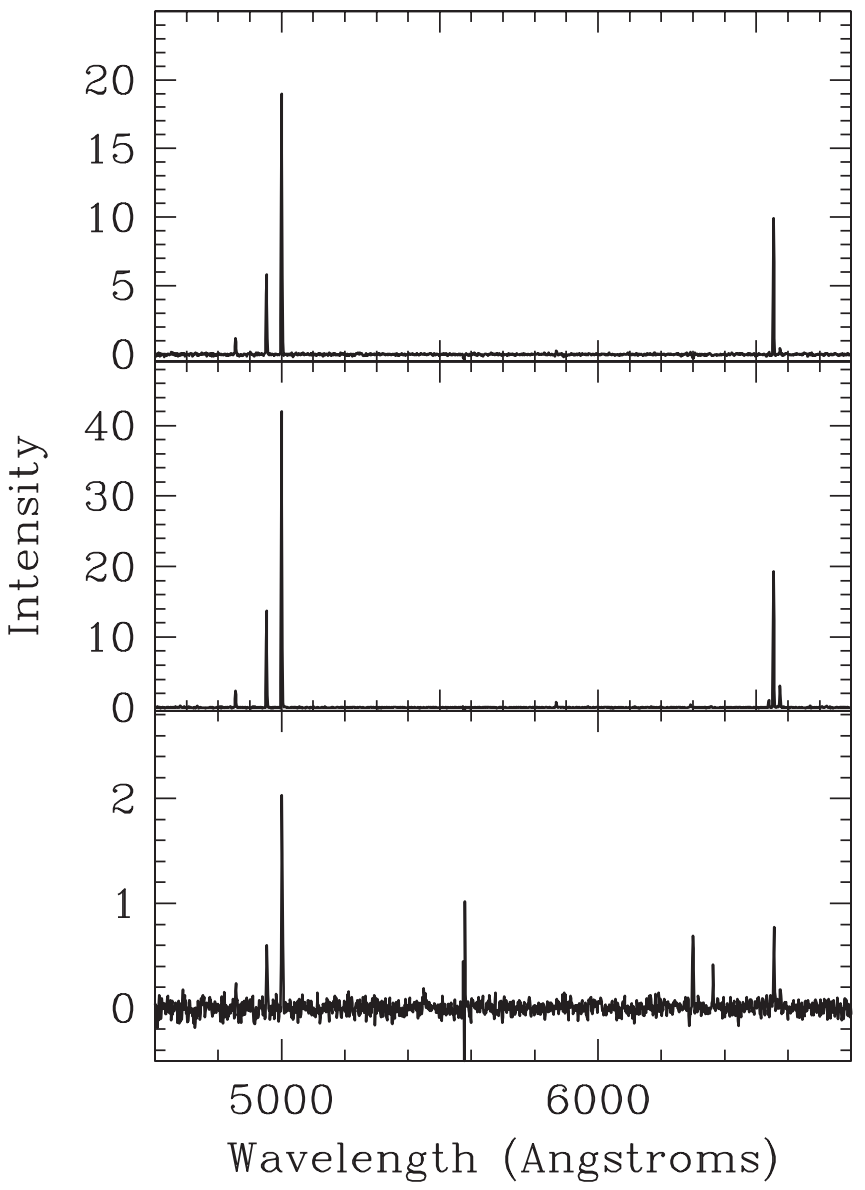

FIG. 4.- Three typical spectra for our sample, with a range of signal-tonoise ratio, showing the strongest PN emission lines, [O III] $\lambda 4959$ and $\lambda 5007$, and $\mathrm{H} \alpha$.

IRAF routines. The individual fiber spectra were extracted using the fiber apertures determined from the dome flats. We then co-added spectra from the individual exposures to make the single deep spectrum for each object.

Highly accurate velocities depend on careful wavelength calibration of the spectra. We used the adjacent $\mathrm{Cu}-\mathrm{Ar}$ lamp exposure for each setup to wavelength calibrate the individual spectra. A fifth-order Legendre function was used to fit 6070 lines in the $\mathrm{Cu}$-Ar spectrum to determine the spectral solution. The rms error in the fits was always less than $0.2 \AA$, or $10 \mathrm{~km} \mathrm{~s}^{-1}$. The bench-mounted spectrograph minimizes the spectral shift. We confirmed this by cross-correlating the comparison lamp spectra from different pointings and found that the spectra shifted by less than $0.05 \AA$ over the course of the night. Figure 4 shows three typical spectra from our sample.

Unlike studies of PN kinematics in distant galaxies where only [O III] $\lambda 5007$ is detected (e.g., Hui et al. 1995; Arnaboldi et al. 1998; Méndez et al. 2001), the proximity of M31 PNs allows us to easily detect $\lambda 5007$ and $\lambda 4959$ in nearly all of our candidates, and $\mathrm{H} \beta$ in most. Local Group PN studies that have used all three lines independently (e.g., Nolthenius \& Ford 1987; Ford et al. 1977) have found that they lead to systematically different velocities. We opt instead to use all three lines simultaneously, thus improving the accuracy of our derived radial velocities.

We used the $r v$ package in IRAF to derive radial velocities using the cross-correlation of the object spectra with a template. Initially, we created an artificial PN spectrum to serve as the 
TABLE 1

Coordinates and Heliocentric Velocities for PN Candidates in M31

\begin{tabular}{|c|c|c|c|c|}
\hline ID & R.A. (2000.0) & Decl. (2000.0) & $v_{\text {helio }}$ & $v_{\text {err }}$ \\
\hline HKPN $1 \ldots \ldots \ldots$ & 003841.29 & 394740.1 & -532.3 & 4.0 \\
\hline HKPN $2 \ldots \ldots \ldots$ & 003844.19 & 401758.9 & -452.8 & 5.1 \\
\hline HKPN $3 \ldots \ldots \ldots$ & 003854.97 & 401016.2 & -362.1 & 1.9 \\
\hline HKPN 4 ......... & 003856.63 & 394714.2 & -425.1 & 12.6 \\
\hline HKPN $5 .$. & 003902.52 & 402250.5 & -511.2 & 3.1 \\
\hline HKPN 6 ......... & 003903.40 & 394558.4 & -484.0 & 3.4 \\
\hline HKPN 7 ......... & 003903.76 & 395329.0 & -477.9 & 3.5 \\
\hline HKPN $8 \ldots \ldots \ldots$. & 003904.46 & 403430.3 & -512.3 & 3.0 \\
\hline HKPN 9 ......... & 003905.33 & 404146.0 & -378.6 & 5.3 \\
\hline HKPN $10 \ldots \ldots$. & 003906.60 & 401459.8 & -548.2 & 4.3 \\
\hline HKPN $11 \ldots \ldots$. & 003909.32 & 401121.0 & -428.2 & 2.5 \\
\hline HKPN $12 \ldots \ldots$. & 003914.80 & 402428.6 & -325.5 & 6.4 \\
\hline HKPN $13 \ldots \ldots$. & 003915.00 & 402634.5 & -522.1 & 5.9 \\
\hline HKPN $14 \ldots \ldots .$. & 003915.80 & 401238.5 & -470.1 & 4.5 \\
\hline HKPN 15 ....... & 003915.84 & 401239.3 & -482.9 & 5.3 \\
\hline HKPN $16 \ldots \ldots .$. & 003916.29 & 402212.8 & -526.1 & 5.6 \\
\hline HKPN 17 ....... & 003918.51 & 400919.0 & -376.3 & 4.1 \\
\hline HKPN $18 \ldots \ldots .$. & 003924.53 & 400702.4 & -434.4 & 2.7 \\
\hline HKPN $19 \ldots \ldots$. & 003926.12 & 404426.0 & -373.1 & 27.7 \\
\hline HKPN $20 \ldots \ldots .$. & 003927.26 & 402903.6 & -532.4 & 12.6 \\
\hline HKPN $21 \ldots \ldots$. & 003931.62 & 401419.9 & -510.4 & 3.6 \\
\hline HKPN $22 \ldots \ldots .$. & 003936.66 & 401751.3 & -503.8 & 3.4 \\
\hline HKPN $23 \ldots \ldots .$. & 003938.40 & 392145.6 & -394.5 & 8.8 \\
\hline HKPN $24 \ldots \ldots .$. & 003940.38 & 401324.7 & -454.4 & 3.1 \\
\hline HKPN 25 ....... & 003942.80 & 402249.2 & -490.9 & 8.0 \\
\hline HKPN $26 \ldots \ldots .$. & 003943.99 & 403542.0 & -465.7 & 12.1 \\
\hline HKPN 27 ....... & 003944.64 & 401959.2 & -500.1 & 3.1 \\
\hline HKPN $28 \ldots \ldots .$. & 003944.79 & 401204.2 & -472.0 & 6.5 \\
\hline HKPN 29 ....... & 003945.40 & 403142.6 & -538.9 & 4.6 \\
\hline HKPN $30 \ldots \ldots$. & 003946.14 & 401101.7 & -416.1 & 2.1 \\
\hline HKPN $31 \ldots \ldots .$. & 003946.71 & 393842.6 & -573.1 & 3.2 \\
\hline HKPN $32 \ldots \ldots$. & 003949.08 & 404239.5 & -168.8 & 28.6 \\
\hline HKPN $33 \ldots \ldots .$. & 003949.62 & 402125.1 & -396.2 & 4.0 \\
\hline HKPN $34 \ldots \ldots .$. & 003952.56 & $\begin{array}{lll}40 & 09 & 32.4\end{array}$ & -481.1 & 6.4 \\
\hline HKPN $35 \ldots \ldots .$. & 003953.93 & 403156.5 & -442.7 & 24.6 \\
\hline HKPN $36 \ldots \ldots .$. & 003954.23 & 395100.0 & -486.0 & 3.3 \\
\hline HKPN 37 ....... & 004001.81 & 403728.7 & -552.4 & 10.6 \\
\hline HKPN $38 \ldots \ldots .$. & 004007.65 & 402834.1 & -457.4 & 5.3 \\
\hline HKPN 39 ........ & 004012.82 & 401302.4 & -387.3 & 4.3 \\
\hline HKPN $40 \ldots \ldots$. & 004013.97 & $40 \quad 1026.4$ & -504.0 & 2.3 \\
\hline HKPN $41 \ldots \ldots .$. & 004014.24 & 402323.9 & -126.1 & 2.5 \\
\hline HKPN $42 \ldots \ldots .$. & 004014.51 & 403850.7 & -513.4 & 12.2 \\
\hline HKPN $43 \ldots \ldots .$. & 004015.29 & 402413.6 & -472.9 & 4.4 \\
\hline HKPN $44 \ldots \ldots .$. & 004016.20 & 400959.7 & -447.9 & 6.9 \\
\hline HKPN $45 \ldots \ldots .$. & 004020.85 & 402808.9 & -506.0 & 2.9 \\
\hline HKPN $46 \ldots \ldots .$. & 004026.61 & 402759.3 & -472.2 & 26.9 \\
\hline HKPN 47 ....... & 004030.80 & 403654.4 & -482.6 & 2.6 \\
\hline HKPN $48 \ldots \ldots .$. & 004033.70 & 401346.8 & -295.0 & 4.7 \\
\hline HKPN 49 ....... & 004037.96 & 401335.5 & -578.9 & 3.3 \\
\hline HKPN $50 \ldots \ldots .$. & 004043.39 & 395702.3 & -423.4 & 2.7 \\
\hline HKPN $51 \ldots \ldots .$. & 004044.02 & 402508.7 & -528.2 & 36.7 \\
\hline HKPN $52 \ldots \ldots .$. & 004044.24 & 402711.2 & -485.8 & 6.1 \\
\hline HKPN $53 \ldots \ldots .$. & 004045.33 & 404130.3 & -524.9 & 5.1 \\
\hline HKPN $54 \ldots \ldots .$. & 004046.82 & 395148.5 & -418.1 & 6.9 \\
\hline HKPN $55 \ldots \ldots .$. & 004047.30 & 402825.6 & -479.4 & 7.7 \\
\hline HKPN $56 \ldots \ldots .$. & 004049.58 & 403946.8 & -416.1 & 18.7 \\
\hline HKPN 57 ....... & 004056.88 & 402009.0 & -560.5 & 3.9 \\
\hline HKPN $58 \ldots \ldots . .$. & 004057.23 & 403607.8 & -498.2 & 8.6 \\
\hline HKPN 59 ....... & 004100.28 & 402850.0 & -425.2 & 2.4 \\
\hline HKPN $60 \ldots \ldots .$. & 004101.86 & 402420.3 & -421.1 & 23.5 \\
\hline HKPN $61 \ldots \ldots .$. & 004103.72 & 404113.6 & -556.1 & 6.0 \\
\hline HKPN $62 \ldots \ldots .$. & $0041 \quad 12.34$ & 403558.2 & -528.4 & 10.0 \\
\hline HKPN 63 ....... & 004113.50 & 404215.8 & -526.7 & 14.0 \\
\hline HKPN 64. & 004120.40 & 392810.6 & -274.0 & 13.8 \\
\hline
\end{tabular}

TABLE 1-Continued

\begin{tabular}{|c|c|c|c|c|}
\hline ID & R.A. (2000.0) & Decl. (2000.0) & $v_{\text {helio }}$ & $v_{\text {err }}$ \\
\hline HKPN 65 ....... & 004126.34 & 395948.4 & -448.2 & 17.7 \\
\hline HKPN $66 \ldots \ldots .$. & 004126.48 & 403245.4 & -399.6 & 4.0 \\
\hline HKPN 67 ........ & 004126.84 & $4043 \quad 16.2$ & -394.0 & 14.1 \\
\hline HKPN 68 ....... & 004133.67 & 402039.5 & -396.6 & 3.8 \\
\hline HKPN 69 ....... & 004138.18 & 395459.8 & -413.5 & 2.3 \\
\hline HKPN $70 \ldots \ldots .$. & 004203.33 & 403139.8 & -539.0 & 4.0 \\
\hline HKPN $71 \ldots \ldots .$. & 004204.07 & 402937.7 & -513.8 & 26.7 \\
\hline HKPN $72 \ldots \ldots .$. & 004205.09 & $4043 \quad 16.3$ & -449.4 & 44.3 \\
\hline HKPN 73 ....... & 004219.12 & 405709.0 & -281.5 & 8.7 \\
\hline HKPN $74 \ldots \ldots .$. & 004221.19 & 403349.9 & -379.7 & 4.2 \\
\hline HKPN $75 \ldots \ldots .$. & 004222.82 & 404306.6 & -509.1 & 6.9 \\
\hline HKPN $76 \ldots \ldots .$. & 004229.75 & $\begin{array}{lll}41 & 03 & 30.4\end{array}$ & -451.3 & 5.4 \\
\hline HKPN 77 ....... & 004232.35 & 404226.2 & -362.2 & 8.6 \\
\hline HKPN $78 \ldots \ldots .$. & 004237.21 & $\begin{array}{lll}41 & 04 & 19.4\end{array}$ & -461.1 & 8.0 \\
\hline HKPN 79 ....... & 004244.49 & 401731.0 & -458.9 & 3.7 \\
\hline HKPN $80 \ldots \ldots .$. & 004246.60 & 402615.2 & -462.8 & 3.9 \\
\hline HKPN $81 \ldots \ldots .$. & 004249.84 & 405110.6 & -505.7 & 10.0 \\
\hline HKPN $82 \ldots \ldots .$. & 004250.58 & 400448.5 & -477.6 & 4.5 \\
\hline HKPN 83 ....... & 004252.05 & 402903.1 & -261.1 & 20.1 \\
\hline HKPN $84 \ldots \ldots .$. & 004252.11 & 402903.9 & -294.1 & 2.6 \\
\hline HKPN $85 \ldots \ldots .$. & 004255.60 & $\begin{array}{lll}41 & 03 & 40.9\end{array}$ & -599.1 & 5.6 \\
\hline HKPN $86 \ldots \ldots .$. & 004256.02 & 405112.5 & -638.3 & 5.3 \\
\hline HKPN 87 ....... & 004256.18 & 403540.4 & -534.8 & 8.6 \\
\hline HKPN $88 \ldots \ldots .$. & 004257.81 & $41 \quad 1243.4$ & -199.2 & 18.0 \\
\hline HKPN 89 ....... & 004258.82 & 412213.2 & -142.5 & 4.6 \\
\hline HKPN $90 \ldots \ldots .$. & 004303.15 & 405718.5 & -367.4 & 11.0 \\
\hline HKPN $91 \ldots \ldots$. & 004305.26 & 405334.1 & -532.3 & 4.8 \\
\hline HKPN 92 ....... & 004306.16 & $41 \quad 1056.0$ & -337.9 & 3.9 \\
\hline HKPN 93 ........ & 004313.72 & 405232.4 & -381.5 & 6.0 \\
\hline HKPN $94 \ldots \ldots .$. & 004315.18 & 405112.7 & -500.3 & 6.2 \\
\hline HKPN 95 ....... & 004319.00 & 405802.1 & -452.0 & 4.3 \\
\hline HKPN 96 ........ & 004320.61 & 405532.4 & -309.9 & 11.2 \\
\hline HKPN 97 ....... & 004320.63 & 405744.5 & -502.8 & 12.0 \\
\hline HKPN $98 \ldots \ldots .$. & 004321.64 & 405912.7 & -388.8 & 28.8 \\
\hline HKPN 99 ........ & 004323.00 & $41 \quad 1540.4$ & -251.3 & 10.0 \\
\hline HKPN $100 \ldots .$. & 004328.32 & 405119.9 & -433.9 & 25.2 \\
\hline HKPN $101 \ldots .$. & 004336.79 & $\begin{array}{lll}41 & 03 & 33.4\end{array}$ & -260.3 & 7.4 \\
\hline HKPN $102 \ldots .$. & 004336.80 & 403738.6 & -323.9 & 12.4 \\
\hline HKPN $103 \ldots . .$. & 004344.52 & 405959.7 & -274.7 & 4.3 \\
\hline HKPN $104 \ldots . .$. & 004404.65 & $41 \quad 1208.9$ & -293.0 & 4.7 \\
\hline HKPN $105 \ldots . .$. & 004404.94 & 410918.6 & -212.8 & 13.7 \\
\hline HKPN $106 \ldots . .$. & 004407.49 & $\begin{array}{lll}41 & 10 & 09.7\end{array}$ & -270.6 & 6.4 \\
\hline HKPN $107 \ldots .$. & 004408.01 & 400313.6 & -391.4 & 3.8 \\
\hline HKPN $108 \ldots .$. & 004411.42 & $\begin{array}{lll}41 & 01 & 02.2\end{array}$ & -348.6 & 6.0 \\
\hline HKPN 109 ..... & 004411.92 & $41 \quad 1400.0$ & -232.4 & 4.5 \\
\hline HKPN $110 \ldots .$. & 004415.88 & 412210.4 & -251.2 & 5.0 \\
\hline HKPN $111 \ldots \ldots$. & 004421.04 & $41 \quad 1541.1$ & -281.8 & 4.2 \\
\hline HKPN $112 \ldots .$. & 004425.50 & 401436.6 & -428.5 & 8.0 \\
\hline HKPN $113 \ldots . .$. & 004425.98 & $40 \quad 1545.7$ & -350.4 & 3.2 \\
\hline HKPN $114 \ldots .$. & 004430.33 & 400729.5 & -425.7 & 2.3 \\
\hline HKPN $115 \ldots .$. & 004437.91 & $41 \quad 1414.4$ & -219.3 & 15.0 \\
\hline HKPN $116 \ldots . .$. & 004437.97 & $\begin{array}{lll}41 & 0702.9\end{array}$ & -335.9 & 11.7 \\
\hline HKPN 117 ..... & 004442.22 & $\begin{array}{lll}41 & 08 & 01.8\end{array}$ & -243.5 & 4.9 \\
\hline HKPN $118 \ldots .$. & 004442.77 & 404005.7 & -383.8 & 4.7 \\
\hline HKPN $119 \ldots .$. & 004443.80 & 411428.0 & -315.7 & 5.9 \\
\hline HKPN $120 \ldots .$. & 004450.69 & 411722.3 & -288.6 & 5.3 \\
\hline HKPN $121 \ldots .$. & 004453.16 & $41 \quad 1558.0$ & -306.6 & 28.5 \\
\hline HKPN $122 \ldots .$. & 004454.43 & $4052 \quad 45.4$ & -453.7 & 3.8 \\
\hline HKPN $123 \ldots .$. & 004454.98 & $\begin{array}{lll}41 & 09 & 53.2\end{array}$ & -321.1 & 5.1 \\
\hline HKPN $124 \ldots .$. & 004456.30 & 412025.4 & -296.6 & 4.5 \\
\hline HKPN $125 \ldots .$. & 004505.05 & $41 \quad 1252.5$ & -201.0 & 10.9 \\
\hline HKPN $126 \ldots .$. & 004505.30 & 410938.6 & -278.5 & 6.3 \\
\hline HKPN $127 \ldots .$. & 004507.14 & 403933.4 & -285.8 & 7.7 \\
\hline HKPN $128 \ldots .$. & 004528.36 & 410454.2 & -400.5 & 16.8 \\
\hline HKPN $129 \ldots .$. & 004535.32 & 405957.2 & -205.1 & 9.4 \\
\hline
\end{tabular}


TABLE 1-Continued

\begin{tabular}{ccccr}
\hline \hline ID & R.A. (2000.0) & Decl. (2000.0) & $v_{\text {heli }}$ & $v_{\text {err }}$ \\
\hline HKPN 130 .... & 004538.13 & 405919.5 & -279.0 & 10.9 \\
HKPN 131 .... & 004700.98 & 404923.4 & -350.5 & 3.1 \\
HKPN 132 .... & 004822.14 & 404541.9 & -320.0 & 3.4 \\
HKPN 133 .... & 004824.78 & 410811.1 & -353.2 & 3.3 \\
HKPN 134 .... & 004827.23 & 395534.0 & -186.8 & 2.4 \\
HKPN 135 .... & 004928.30 & 405954.0 & -242.6 & 3.4 \\
\hline
\end{tabular}

Note.- Units of right ascension are hours, minutes, and seconds, and units of declination are degrees, arcminutes, and arcseconds.

template spectrum (three lines matched to the instrumental PSF at $\lambda 5007, \lambda 4959$, and $\mathrm{H} \beta$ ), but we found that we were able to reduce the internal velocity errors significantly by using a spectrum created by combining real PN spectra as a template. We selected the eight highest signal-to-noise ratio spectra from the PNs in the first pointing. After continuum-subtracting them, we shifted them to zero velocity by fitting a Gaussian to the $\lambda 5007$ line profile. The final template was created by co-adding these eight spectra. The radial velocities are derived by calculating the cross-correlation function of the template and continuumsubtracted PN spectra in the spectral region including 25007 , 24959 , and $\mathrm{H} \beta$, using fxcor in IRAF. In general, we did not sky subtract the PN spectra because there were no significant sky lines or stellar absorption lines in this spectral region. The resulting radial velocities and errors are listed in Table 1.

\subsection{Velocity Errors}

All the PNs were observed on the same night with the same instrument, making systematic differences within the PN sample highly unlikely. We observed 11 PNs from our sample in multiple pointings. The mean difference in velocity for these PNs is $-1.2 \mathrm{~km} \mathrm{~s}^{-1}$, with a standard deviation of $4 \mathrm{~km} \mathrm{~s}^{-1}$. These results are consistent with the typical internal velocity error estimates from fxcor of 3-7 $\mathrm{km} \mathrm{s}^{-1}$. We estimate an additional $5 \mathrm{~km} \mathrm{~s}^{-1}$ of error in the velocities because of systematics effects.

There have been several earlier studies of emission-line objects in or near M31, and this allows us to assess the systematic error in our velocities. We have spectra for three H II regions in the dwarf galaxy And IV (objects 3, 4, and 6 from Ferguson et al. 2000). The velocities for objects 4 and 6 agree within $5 \mathrm{~km} \mathrm{~s}^{-1}(250 \pm 5.2$ and $270.8 \pm 39.2$, respectively, compared to 250 and $273 \mathrm{~km} \mathrm{~s}^{-1}$ from Ferguson et al. 2000). For object 3, we find a velocity of $233.0 \pm 3.4 \mathrm{~km} \mathrm{~s}^{-1}$, compared to $244 \mathrm{~km} \mathrm{~s}^{-1}$, a difference of $11 \mathrm{~km} \mathrm{~s}^{-1}$. Our sample and that of NF have 11 PNs in common. The mean difference $v_{\text {this }}-v_{\mathrm{NF} 87}$ is $10.4 \mathrm{~km} \mathrm{~s}^{-1}$. This is the same systematic difference that NF87 note between their velocities and those of Ford et al. (1977) for the Galactic PN velocity standards used in the two studies. Given the good agreement between our velocities and those of Ferguson et al. (2000) and the disagreement between the NF87 and Ford et al. (1977) velocities, we conclude that there is a systematic offset in the NF87 velocities. In the remaining discussion, we have offset their velocities by $+10.4 \mathrm{~km} \mathrm{~s}^{-1}$ to agree with ours.

\section{RESULTS}

\subsection{Overview of PN Kinematics}

Although M31 is the primary target of our survey, M32 and the dwarf galaxy And IV are also located in the survey region.
TABLE 2

PN Candidates in M32

\begin{tabular}{|c|c|c|c|c|}
\hline ID & R.A. (2000.0) & Decl. (2000.0) & $v_{\text {helio }}$ & $v_{\text {err }}$ \\
\hline 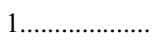 & 004223.24 & 404628.7 & -182.4 & 8.4 \\
\hline $2 \ldots \ldots \ldots \ldots \ldots$ & 004228.93 & 404439.0 & -158.3 & 6.6 \\
\hline 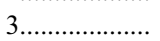 & 004240.10 & 404941.3 & -160.5 & 3.6 \\
\hline 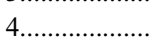 & 004240.24 & 405103.0 & -149.6 & 2.3 \\
\hline 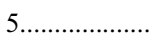 & 004250.63 & 404528.4 & -183.7 & 2.3 \\
\hline 6 & 004253.02 & 404859.8 & -213.5 & 2.0 \\
\hline 7.․․․․…... & 004256.97 & 405101.8 & -169.1 & 24.8 \\
\hline 8 & 004302.04 & 404930.2 & -154.6 & 4.7 \\
\hline
\end{tabular}

Note.-Units of right ascension are hours, minutes, and seconds, and units of declination are degrees, arcminutes, and arcseconds.

Membership for several objects is ambiguous and was decided on the basis of position and velocity.

The three emission-line objects we identified in And IV are H II regions, resolved in Hubble Space Telescope images (Ferguson et al. 2000). Although PNs commonly have $\xi=$ [O III] $\lambda 5007 / \mathrm{H} \alpha \sim 3$, values nearer to 1 are possible, so $\xi$ for PNs and high-excitation $\mathrm{H}$ II regions overlap significantly. This ambiguity prevents us from distinguishing PNs from $\mathrm{H}$ II regions solely with our spectra; such a distinction requires nebular modeling, which relies on faint lines beyond our wavelength range. The inclusion of the And IV H II regions in our sample raises the question of substantial numbers of M31 H II regions in the sample. However, this is unlikely for several reasons. The And IV H II region spectra have $\xi \sim \frac{1}{3}$, much less than the rest of our sample. And IV is significantly more distant than M31 ( $>5$ Mpc; Ferguson et al. 2000). Bright M31 H II regions are more likely to be resolved, and we considered only point sources as candidates. Also, although the value of $\xi$ ranged from 1 to 3 (excluding the And IV $\mathrm{H}$ II regions), objects with values as low as 1 are rare in our sample $(<10 \%)$. With this in mind, we refer to the emission-line objects in M31 as PNs throughout the rest of the discussion.

M32 PNs are clumped in position and in velocity near M32's radial velocity of $-197 \mathrm{~km} \mathrm{~s}^{-1}$ (Huchra et al. 1999). For M32, $\sigma_{v} \sim 50 \mathrm{~km} \mathrm{~s}^{-1}$, and the tidal radius is $\sim 2 \mathrm{kpc}$ (Mateo 1998). Nine PNs in the sample have velocities within $50 \mathrm{~km} \mathrm{~s}^{-1}$ of M32's systemic velocity and are located within $1 \mathrm{kpc}$ of M32's center. PN membership is summarized in Tables 1, 2, and 3. Each table lists the positions and heliocentric radial velocities for the emission-line objects in M31, M32, and And IV, respectively. We have excluded M32 and And IV objects from the analysis that follows.

The positions of the PNs relative to the center of M31 are shown in Figure 5. We have assumed a distance of $770 \mathrm{kpc}$ to M31 (Freedman \& Madore 1990). $X$ is the distance in the major axis direction, and $Y$ is the distance in the minor axis

TABLE 3

H it Regions in And IV

\begin{tabular}{|c|c|c|c|c|}
\hline $\mathrm{ID}^{\mathrm{a}}$ & R.A. (2000) & Decl. (2000) & $v_{\text {helio }}$ & $v_{\text {err }}$ \\
\hline $6 \ldots$ & 004230.57 & 403446.9 & 270.2 & 39.2 \\
\hline 3........................ & 004232.17 & 403358.7 & 233.0 & 3.4 \\
\hline 4 & 004231.73 & 403411.2 & 249.5 & 5.2 \\
\hline
\end{tabular}

NoTE.- Units of right ascension are hours, minutes, and seconds, and units of declination are degrees, arcminutes, and arcseconds.

${ }^{\mathrm{a}}$ ID numbers correspond to those given in Ferguson et al. (2000). 


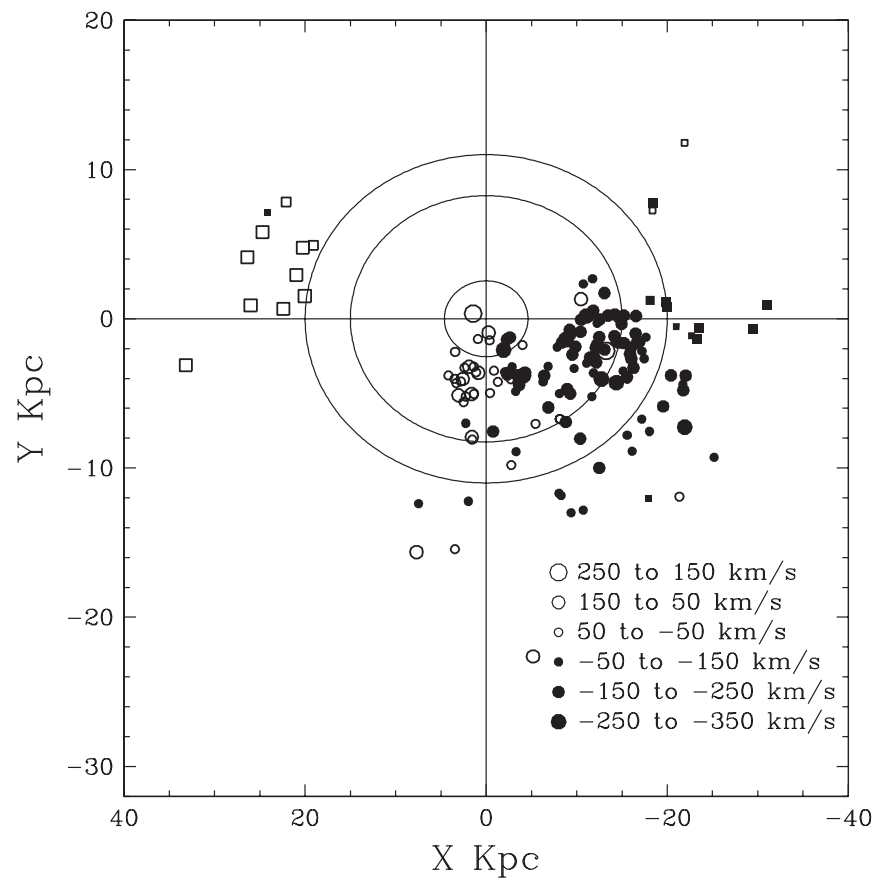

FIG. 5.-Positions of PNs in M31. The major axis has been rotated through the position angle so that it is horizontal in the figure. Symbol size corresponds to velocity. Circles are PN velocities from this study, and squares are those from NF87. Ellipses are bulge isophotes with $b / a=0.55$.

direction. The positions have been rotated through M31's position angle ( $37^{\circ} .3$; de Vaucouleurs 1958$)$ so that the major axis defines $Y=0$. All velocities are plotted relative to M31's systemic velocity and coded by symbol size. We have enlarged our sample of PNs by adding the objects from NF87 that are not already in our sample.

The depth and spatial sampling of the survey is not uniform and must be taken into account when interpreting the spatial distribution of the PNs. Only field 3 intersects the major axis (compare Figs. 2 and 5). Fields 1 and 2 had a sizable gap between them, and this can be seen in Figure 5 around $Y=$ $-12 \mathrm{kpc}$. Fields 1 and 3 are of comparable depth, while field 2 is shallower. Finally, the objects nearest the center of the galaxy, where we did not attempt to identify PNs in the Schmidt images, are PNs from the sample of Hui et al. (1994) used to fill spare fibers, as mentioned previously.

The various populations in M31 can be isolated by splitting the sample in $Y$. Figure 6 shows plots of distance along the major axis versus the line-of-sight velocity for several ranges of $|Y|$. The disk dominates at $|Y|<0.5 \mathrm{kpc}$, where the upper limit on the PN velocities is the $\mathrm{H}$ I rotation curve (Kent 1989). A mixture of disk and bulge objects are seen at $|Y|=0.5-6 \mathrm{kpc}$. Bulge PNs near the minor axis of the galaxy have velocities of -150 to $150 \mathrm{~km} \mathrm{~s}^{-1}$ relative to M31's systemic velocity, consistent with the large bulge central velocity dispersion of $100-$ $150 \mathrm{~km} \mathrm{~s}^{-1}$ (McElroy 1983; Richstone \& Shectman 1980). Some of the highest velocity objects in this region (crosses) may be associated with the newly discovered satellite And VIII (Morrison et al. 2003). We expect to find bulge and halo objects at $|Y|>6 \mathrm{kpc}$. We discuss the distinction between these regions further in $\S 3.2$.

A striking feature of Figure 6 is the very small number of PNs occupying the "forbidden" quadrants for disk objects, even at large $Y$. This lack of objects in the two nondisk quadrants is the signature of a rotationally supported population, which clearly dominates our sample.
Disentangling the various stellar populations in M31 is complicated by the galaxy's inclination ( $77^{\circ}$ from face-on), which causes objects in the outer disk to appear in projection away from the major axis. Disk, bulge, and halo populations therefore overlap spatially and kinematically. The combination of position and velocity data, however, offers the potential to isolate the different populations. The allowed velocities for a given PN position will depend on the kinematic population to which it belongs. In the discussion that follows, we present an overview of the expected spatial and kinematic signatures of the disk and bulge, then use simple kinematic models to distinguish between them, as well as identify halo candidates. We adopt the following definitions: $v_{\text {los }}$ and $\sigma_{\text {los }}$ are the observed, line-of-sight velocity and dispersion, respectively; for a given population, $v_{\text {rot }}$ is the rotation velocity and $\sigma_{r}$ is the radial velocity dispersion; and $v_{\text {circ }}$ is the circular velocity of the disk, which reflects the mass distribution, and which we take to be the $\mathrm{H}$ I $v_{\text {rot }}$, roughly $250 \mathrm{~km} \mathrm{~s}^{-1}$.

\subsection{M31's Inclined Disk \\ 3.2.1. Spatial Extent of the Disk}

The spatial signature of the disk will depend on the size and thickness of the disk, as well as the degree of warping and the radius at which the warp begins. However, we can draw some simple conclusions from basic geometry. The radial extent of the optical stellar disk was estimated by Walterbos \& Kennicutt (1988, hereafter WK88) to be $26 \mathrm{kpc}$. There are now two very deep star count images that can be used to check this result, from Ferguson et al. (2002) and Zucker et al. (2004). These images reach to on the order of $30 \mathrm{~V}$ mag $\operatorname{arcsec}^{-2}$ and are thus very sensitive tests for the extent of the disk. In both images it can be seen that the brighter portions of the outer disk extend to the WK88 value, that there are some fainter regions that extend to $R=35 \mathrm{kpc}$ and may be associated with tidal streams or with the outer disk, and that there is no starlight beyond $35 \mathrm{kpc}$ to very sensitive limits. We have thus assumed $R_{\max }=26 \mathrm{kpc}$ and consider the effect of a larger $R_{\max }$ when relevant in the discussion that follows.

If the disk were planar and nonwarped, its edge would project to a distance of $5.6 \mathrm{kpc}$ from the major axis because of M31's inclination. Near the minor axis, the disk and bulge contribute roughly equally to the luminosity from 2 to $5 \mathrm{kpc}$ (see Fig. 3 of WK88). We can therefore expect the bulge and halo PNs to dominate beyond $\sim 5 \mathrm{kpc}$ from the major axis. These rough geometrical arguments are supported by observations. On the side of the major axis covered by our survey, the outer optical isophotes of the galaxy $\left(\mu_{B} \sim 26\right)$ extend $4.4-$ $6.6 \mathrm{kpc}\left(20^{\prime}-30^{\prime}\right)$ from the major axis (WK88); $\mathrm{H}$ il regions are found up to $4 \mathrm{kpc}$ (Pellet et al. 1978).

However, the assumption of a perfectly planar disk is not justified. Neutral hydrogen studies (Roberts \& Whitehurst 1975; Cram et al. 1980; Brinks \& Shane 1984) have found two distinct velocity systems for many lines of sight through M31's disk. This suggests that the line of sight is crossing the disk twice. Brinks \& Burton (1984) interpreted the dual kinematics as a warp signature. In their model, the geometry of the galaxy is such that the line of nodes is nearly aligned with the major axis and the warp bends over back toward the major axis on the near (northwest) side, so that the warp is nearly edge-on (Brinks \& Burton 1984, Fig. 2a). The unwarped H I has a radius of $16 \mathrm{kpc}$, which would project to a height of $4 \mathrm{kpc}$ from the major axis.

Brinks \& Burton (1984) introduce a flare of increasing scale height in their model of the $\mathrm{H}$ I warp. The flare crosses the line 


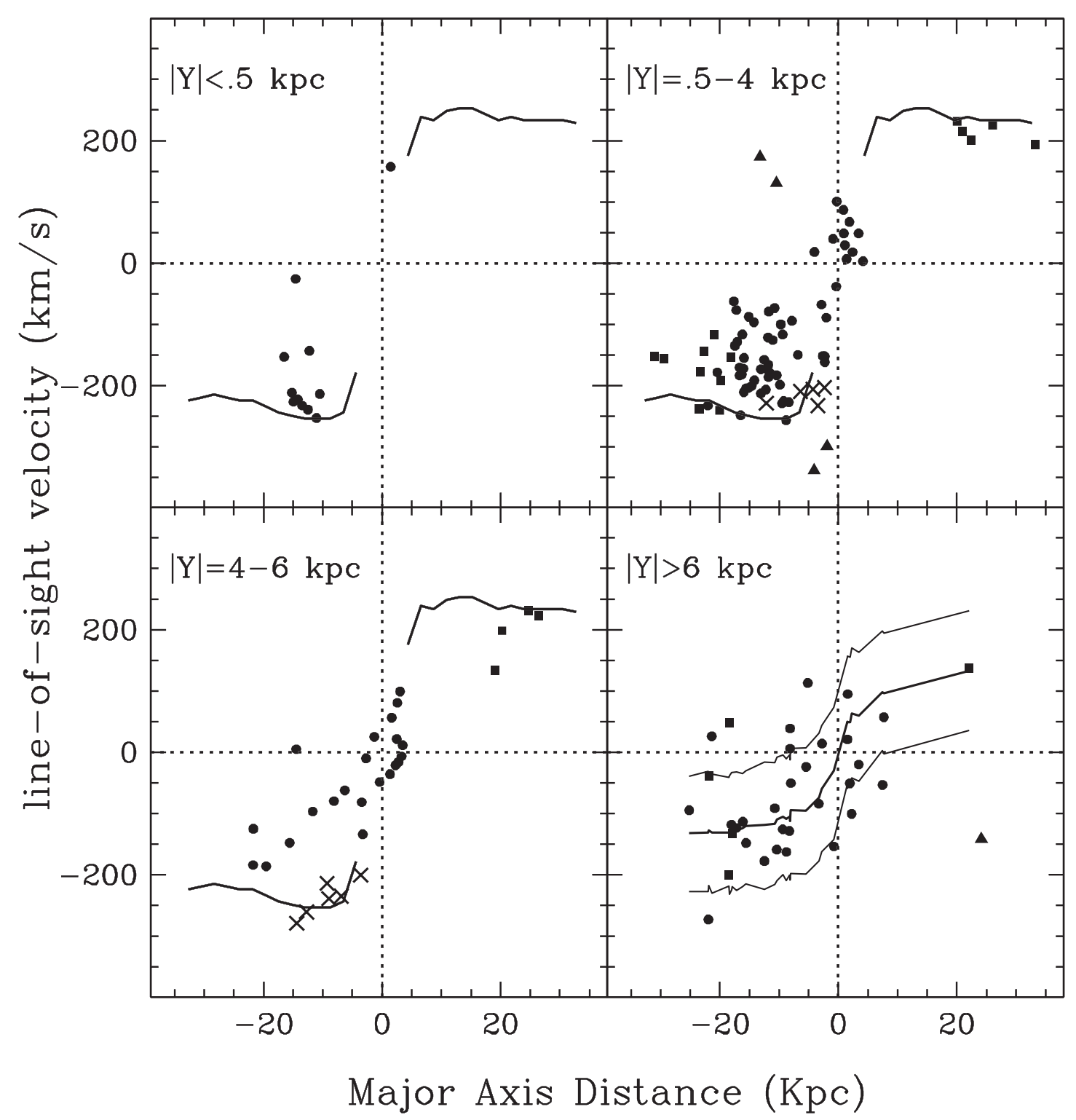

Fig. 6.-Velocity-distance diagram for PNs in M31. $X$ is distance along the major axis; $Y$ is the projected distance in the direction of the minor axis. Filled circles (PNs from our survey) and squares (PNs from NF87) are PNs with M31 disk or bulge velocities; triangles are halo candidates; crosses are probable And VIII members (Morrison et al. 2003). The rotation curve is taken from Kent (1989). The solid lines in the lower right panel represent the range of values that could be expected for the PNs for our best bulge model as discussed in $\S 3.4$. [See the electronic edition of the Journal for a color version of this figure.]

of sight to the major axis and accounts for the two velocity systems there. Flares are common in the outer $\mathrm{H}$ I disks of galaxies (Sancisi \& Allen 1979; Kulkarni et al. 1982; Olling 1996), but the evidence for flared stellar disks is conflicting. Studies of other galaxies have generally found a constant stellar scale height at all radii (van der Kruit \& Searle 1981; Morrison et al. 1994). Recent in situ analyses of Milky Way stars, however, suggest that its stellar disk may be flared (Lopez-Corredoira et al. 2002; Drimmel \& Spergel 2001). Drimmel \& Spergel (2001) find that a flare parameter of $6.6 \mathrm{pc} \mathrm{kpc}^{-1}$ slightly improves their model fits. However, this amounts to an increase of less than $0.25 \mathrm{kpc}$ over the entire length of the disk. This is a relatively small increase and would be indistinguishable from an unflared disk in our data.

Braun (1991) analyzed existing $\mathrm{H}$ I data and concluded that the warp model is too simple. He suggested that the data were better explained by a more complex change in position of the H I disk's midplane, perhaps caused by gravitational perturbations from M32. In this model, the position of the disk midplane varies from $500 \mathrm{pc}$ above the nominal plane to $1 \mathrm{kpc}$ below it (see Braun 1991, Fig. 10). If M31's disk had been disturbed by M32's passage, dynamical evolution may have thickened the stellar disk to an even larger scale height. However, the cold disk kinematics of some globular clusters rules out a major interaction, making a disk thickened in this way unlikely (Morrison et al. 2004). On the basis of these arguments, we expect that the disk's luminous influence should have waned entirely by $Y=5-6 \mathrm{kpc}$.

\subsubsection{Kinematic Signature of the Disk}

The disk's distinctive kinematic signature can be illustrated by first considering a simple edge-on disk with zero velocity dispersion and constant circular velocity; a schematic is shown in Figure 7 . The line of sight through a given major axis distance, $X$, intersects the disk at different radii, resulting in different projections of the rotation velocity, $v_{\text {rot }}$, into the line of 


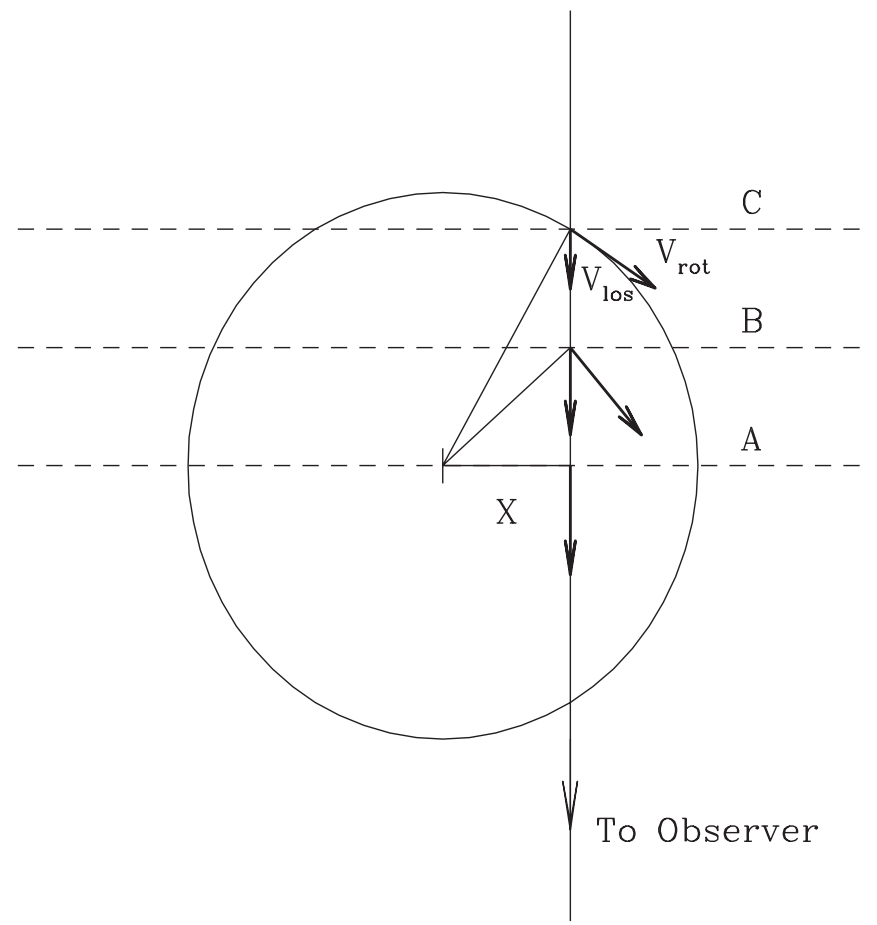

FIg. 7.- Schematic of an edge-on disk, viewed from above. The observer is off to the bottom. The quantity $v_{\text {rot }}$ is the rotation velocity, and $v_{\text {los }}$ is the observed radial velocity. The major axis as observed would lie along line A. The tangent point occurs at A where $v_{\text {los }}=v_{\text {rot }}$.

sight. At a single value of $X$, any velocity between that at $\mathrm{A}$ and that at $\mathrm{C}$ is allowed. The lowest velocity allowed decreases toward the center of the disk, where the line-of-sight velocity, $v_{\text {los }}$, is zero. Corresponding simulated velocity-distance diagrams are shown in Figure 8. Disk objects will be found in only two of the four quadrants of the velocity-distance diagram, corresponding to the approaching and receding sides of the disk.

Because the disk is inclined rather than exactly edge-on, the distance $Y$ from the major axis correlates with position in the disk. For example, lines of sight through the major axis intersect the orbital tangent point (Fig. 8, left); $v_{\text {los }}$ is the component of $v_{\text {rot }}$ projected into the line of sight through the inclination. Lines of sight through the disk between B and C (Fig. 8, right) exclude the major axis points and can have only lower values down to the lowest allowed by the edge of the disk. This basic geometry also holds for a more realistic, hotter disk with some velocity dispersion, although the velocity dispersion tends to smooth out sharp features.

\subsection{Disk PN Kinematics}

PNs near the major axis are those most likely to be disk objects (Fig. 6, upper left). There are $10 \mathrm{PNs}$ with $|Y|<0.5$ and $|X|>10$. One object near $X=-13 \mathrm{kpc}$ has $v_{\text {los }}=-25 \mathrm{~km}$ $\mathrm{s}^{-1}$, more than $200 \mathrm{~km} \mathrm{~s}^{-1}$ from the $\mathrm{H}$ I rotation velocity, and probably belongs to the bulge or halo, so we have excluded it from the disk sample. The nine remaining PNs have an average $v_{\text {los }}$ of $215 \mathrm{~km} \mathrm{~s}^{-1}$, with $\sigma_{\text {los }}=39 \mathrm{~km} \mathrm{~s}^{-1} ;{ }^{6} \sigma_{\text {los }}$ is dominated by the $\sigma_{\phi}$-component of the velocity ellipsoid on the major axis, implying $\sigma_{r} \sim 55 \mathrm{~km} \mathrm{~s}^{-1}$.

\footnotetext{
${ }^{6}$ These values have been corrected for M31's inclination. We have dropped the negative sign indicating rotation toward the observer throughout the remainder of the paper.
}

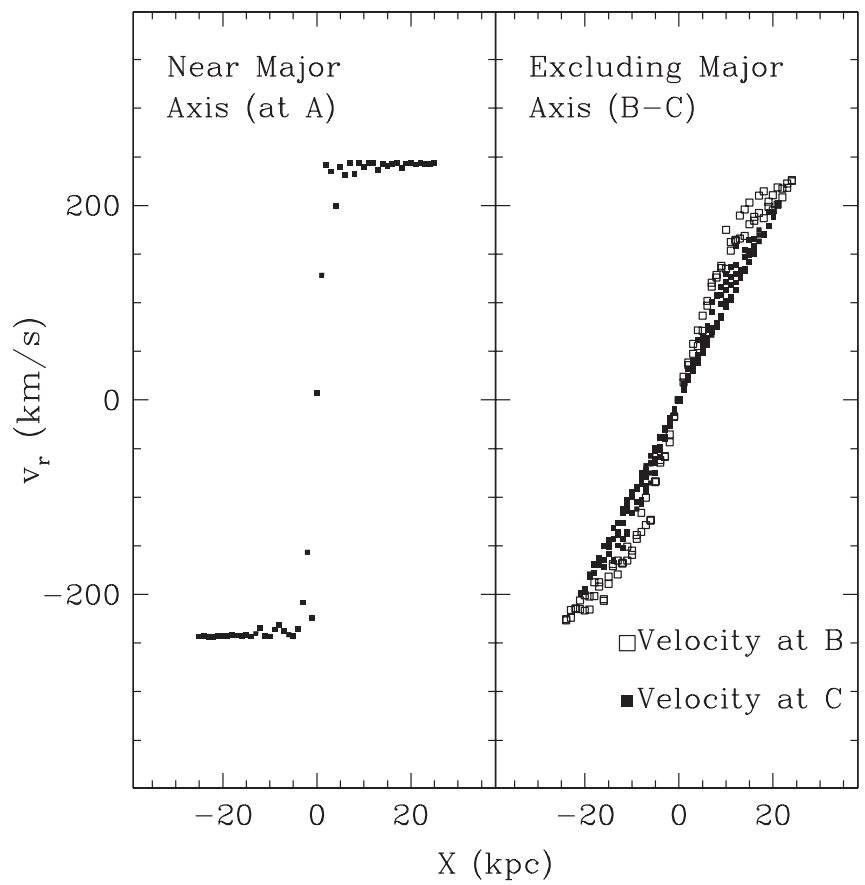

FIG. 8.-Velocities from a model disk, as in Fig. 7, but with M31's $v_{\text {circ }}$ and inclination.

However, these values of $v_{\text {rot }}$ and $\sigma_{r}$ are very uncertain. First, the inclusion of off-axis PNs in the average systematically reduces the measured mean $v_{\text {los }}$. Second, the sample is very small, and the statistics do not take into account the possible inclusion of bulge PNs. At this distance along the major axis, the bulge contributes $\sim 15 \%$ of the total luminosity (WK 88 ), or potentially $1-2$ PNs of the nine in this sample. Two PNs have velocities more than $100 \mathrm{~km} \mathrm{~s}^{-1}$ from the $\mathrm{H}$ I rotation, and removing those increases the average $v_{\text {rot }}$ to $234 \mathrm{~km} \mathrm{~s}^{-1}$ and reduces $\sigma_{r}$ to $20 \mathrm{~km} \mathrm{~s}^{-1}$. Third, the disk rotation velocity is not a secure measurement and is uncertain at the $10-20 \mathrm{~km} \mathrm{~s}^{-1}$ level (see the discussion in Morrison et al. 2004).

With this sample, we cannot distinguish between a thin and thick disk, and more disk PNs are needed (Merrett et al. 2003; D. Hurley-Keller et al. 2004, in preparation). Morrison et al. (2004) show that a large subsample of globular clusters that project onto the disk have thin disk kinematics. This suggests that there has been no merger with M31 large enough to disrupt its thin disk since the formation of the clusters and is compelling evidence against a thick disk in M31.

\subsection{Beyond the Disk: Bulge and Halo}

At $|Y|>6 \mathrm{kpc}$, the PN kinematics is still dominated by rotation (Fig. 6, lower right). The mean $v_{\text {los }}$ of the PNs with $|X|>10$ and $|Y|>6$ is $116 \mathrm{~km} \mathrm{~s}^{-1}$, with $\sigma_{\text {los }}=79 \mathrm{~km} \mathrm{~s}^{-1}$. These PNs clearly do not belong to a high velocity dispersion, nonrotating halo like that of the Milky Way. To what population do they belong?

\subsubsection{Overview of Kinematic Models}

To answer this, we constructed simple kinematic models of disk and bulge populations as described in Morrison et al. (2004). Briefly, for each PN, $X$ and $Y$ are known, but the lineof-sight depth is not. Using a Monte Carlo method, we choose a likely line-of-sight position based on the density distribution and iterate until a detectable PN results. Once we have a 
TABLE 4

Kinematic Models

\begin{tabular}{ccccccc}
\hline \hline Model & $\begin{array}{c}h_{r} \\
(\mathrm{kpc})\end{array}$ & $\begin{array}{c}h_{z} \\
(\mathrm{kpc})\end{array}$ & $\begin{array}{c}v_{\text {rot }} \\
\left(\mathrm{km} \mathrm{s}^{-1}\right)\end{array}$ & Density Distribution & $\begin{array}{c}\sigma_{r} \\
\left(\mathrm{~km} \mathrm{~s}^{-1}\right)\end{array}$ & $\begin{array}{c}\sigma_{\phi}, \sigma_{z} \\
\left(\mathrm{~km} \mathrm{~s}^{-1}\right)\end{array}$ \\
\hline Thin ............ & 5.9 & 0.3 & 230 & $L(R, z)=e^{-R / h_{r}} e^{-z / h_{z}}$ & $\sigma_{r}=\sqrt{h_{z}} v_{\text {circ }} e^{-R / 2 h_{r}}$ & $\left(\sigma_{\phi}, \sigma_{z}\right)=\left(\frac{\sigma_{r}}{\sqrt{2}}, \frac{\sigma_{r}}{2}\right)$ \\
Thick ........... & 5.9 & 1.0 & 230 & $L(R, z)=e^{-R / h_{r}} e^{-z / h_{z}}$ & $\sigma_{r}=\sqrt{h_{z}} v_{\text {circ }} e^{-R / 2 h_{r}}$ & $\left(\sigma_{\phi}, \sigma_{z}\right)=\left(\frac{\sigma_{r}}{\sqrt{2}}, \frac{\sigma_{r}}{2}\right)$ \\
Bulge $\ldots . . . . . . . .$. & 2.4 & $\ldots$ & 150 & $L(R, z)=e^{-7.67\left[\left(-R / R_{e}\right)^{1 / 4}-1\right]}$ & $\sigma_{r}=$ const & $\sigma_{\phi}=\sigma_{z}=\sigma_{r}$ \\
\hline
\end{tabular}

line-of-sight depth, we randomly generate an appropriate velocity for that position in the disk. The velocity is then projected into the line of sight. The result is a predicted velocity for each PN position given a specific kinematic model.

For the thin and thick disks, we assume an exponential surface brightness distribution in $R$ and $z$;

$$
L(R, z)=e^{-R / h_{r}} e^{-z / h_{z}},
$$

where $R$ is the cylindrical radius and $z$ is the height above the galaxy plane.

For disk parameters of scale height, scale length, and maximum radius, we have used the Milky Way and other spiral galaxies as templates. We adopted a scale height $h_{z}$ of $0.3 \mathrm{kpc}$ for the thin disk, comparable to that of the Milky Way thin disk. For the thick disk, we chose a scale height of $1 \mathrm{kpc}$; increasing this to $2 \mathrm{kpc}$ has no effect on our conclusions. The thin and thick disk scale length $h_{r}$ and maximum radius are fixed at 5.9 and $26 \mathrm{kpc}$, respectively, values taken from WK88 and scaled for our assumed distance to M31 of $770 \mathrm{kpc}$. Although thick disks in other galaxies have larger scale lengths than the thin disks (Morrison 1999; Neeser et al. 2002), varying the thick disk scale length by $20 \%$ had no measurable effect on the models we discuss in the next section. Thus far, the few attempts to constrain the cutoff radius of thick disks suggest that thin and thick disks in a given galaxy have the same extent (Pohlen et al. 2004).

At the large values of $X$ under consideration, $v_{\text {rot }}$ can be treated as constant, and we fix $v_{\text {circ }}$ at $250 \mathrm{~km} \mathrm{~s}^{-1}$, a representative value based on the $\mathrm{H}$ I data. We have adopted a relationship between $v_{\text {circ }}$ and $\sigma_{r}$ derived from the van der Kruit \& Freeman (1984) and Bottema (1993) studies of galaxy disks:

$$
\sigma_{r}=\frac{1}{3} v_{\text {circ }} e^{-R / 2 h_{r}} .
$$

This relationship can be normalized to match the Milky Way thin and thick disk values of $\sigma_{r}$ at the solar radius, taken from reviews by Norris (1999) and Morrison (1999) and listed in Table 4 . The equation becomes

$$
\sigma_{r}=\sqrt{h_{z}} v_{\text {circ }} e^{-R / 2 h_{r}} .
$$

The velocity ellipsoid follows from the epicycle approximation (e.g., Binney \& Tremaine 1987):

$$
\left(\sigma_{r}, \sigma_{\phi}, \sigma_{z}\right)=\left(\sigma_{r}, \frac{\sigma_{r}}{\sqrt{2}}, \frac{\sigma_{r}}{2}\right)
$$

For the bulge model, we use an $R^{1 / 4}$ surface brightness profile, with an axial ratio of $b / a=0.55$ (PvdB94), and adopt an isotropic velocity ellipsoid $\left(\sigma_{\phi}=\sigma_{z}=\sigma_{r}\right)$, with constant rotation at these large values of $X$. This simplification is not unreasonable. As yet, little is known about the behavior of bulges at large radii; some bulges exhibit cylindrical rotation, while others show a decrease in $v_{\text {rot }}$ with distance from the midplane (Kormendy \& Illingworth 1982). In the current sample, there are too few PNs to test a more detailed model.

We have chosen an axisymmetric model for the bulge, although some authors suggests that M31's inner bulge is triaxial (Braun 1991; Stark \& Binney 1994; Berman \& Loinard 2002). We note that the 2MASS image of the M31 bulge (Jarrett et al. 2003) shows considerably less isophote twisting than the optical images, suggesting that the disk warp may have contributed to earlier impressions of a triaxial bulge. The $\mathrm{H}$ i rotation curve of Braun (1991) shows a strong central peak, which he attributes to noncircular gas orbits, suggesting a triaxial potential. However, the CO rotation curve of Loinard et al. (1999) has a smaller central peak than the H i curve of Braun (1991). Thus, the bulge may not be strongly triaxial.

\subsubsection{Modeling Results}

Representative thin disk, thick disk, and bulge models for the outer PNs are shown with the data for comparison in Figure 9. The important properties of these models are summarized in Table 5. Only a small fraction of the PNs have positions that can be fitted by the thin disk model. Most of the PNs (20 of 33) at $|Y|>6$ would deproject to radii beyond the optical edge of the disk at 25-35 kpc. Perhaps more convincingly, the PN velocities are also incompatible with this model. Because velocity dispersion falls off with radius in a disk of constant scale height (van der Kruit \& Freeman 1984; Bottema 1993) and is low $\left(\sim 20-30 \mathrm{~km} \mathrm{~s}^{-1}\right)$ for a thin disk at all radii, the disk at this large radius has a low line-of-sight velocity dispersion. This kinematic discrepancy between the model and the data holds regardless of the maximum radius of the model disk used.

The thick disk model could account for the PNs at $|Y|=$ 6-10 kpc; however, the velocity dispersion along the minor axis in the model is significantly less than observed because PNs this far from the major axis would belong to the outer disk where the dispersion is relatively low. By $25 \mathrm{kpc}, \sigma_{r}$ has fallen to less than $40 \mathrm{~km} \mathrm{~s}^{-1}$ in our model of the thick disk. The contribution of the thick disk is vanishingly small by $|Y|=10 \mathrm{kpc}$, and the 13 PNs beyond this (see Fig. 5) are not fitted by the thick disk model. Those 13 PNs have a significant rotation component, with an average $v_{\text {los }}$ of $\sim 70 \mathrm{~km} \mathrm{~s}^{-1}$.

Occam's razor is an appropriate consideration here-there is no need to introduce a new stellar population in M31. The 13 PNs beyond $|Y|=10 \mathrm{kpc}$ already represent a rotating population that are not explained by the thick disk model. No convincing evidence of a thick disk in M31 has yet emerged, and the cold kinematics of many M31 clusters suggests that M31 has not experienced a merger massive enough to form a thick 


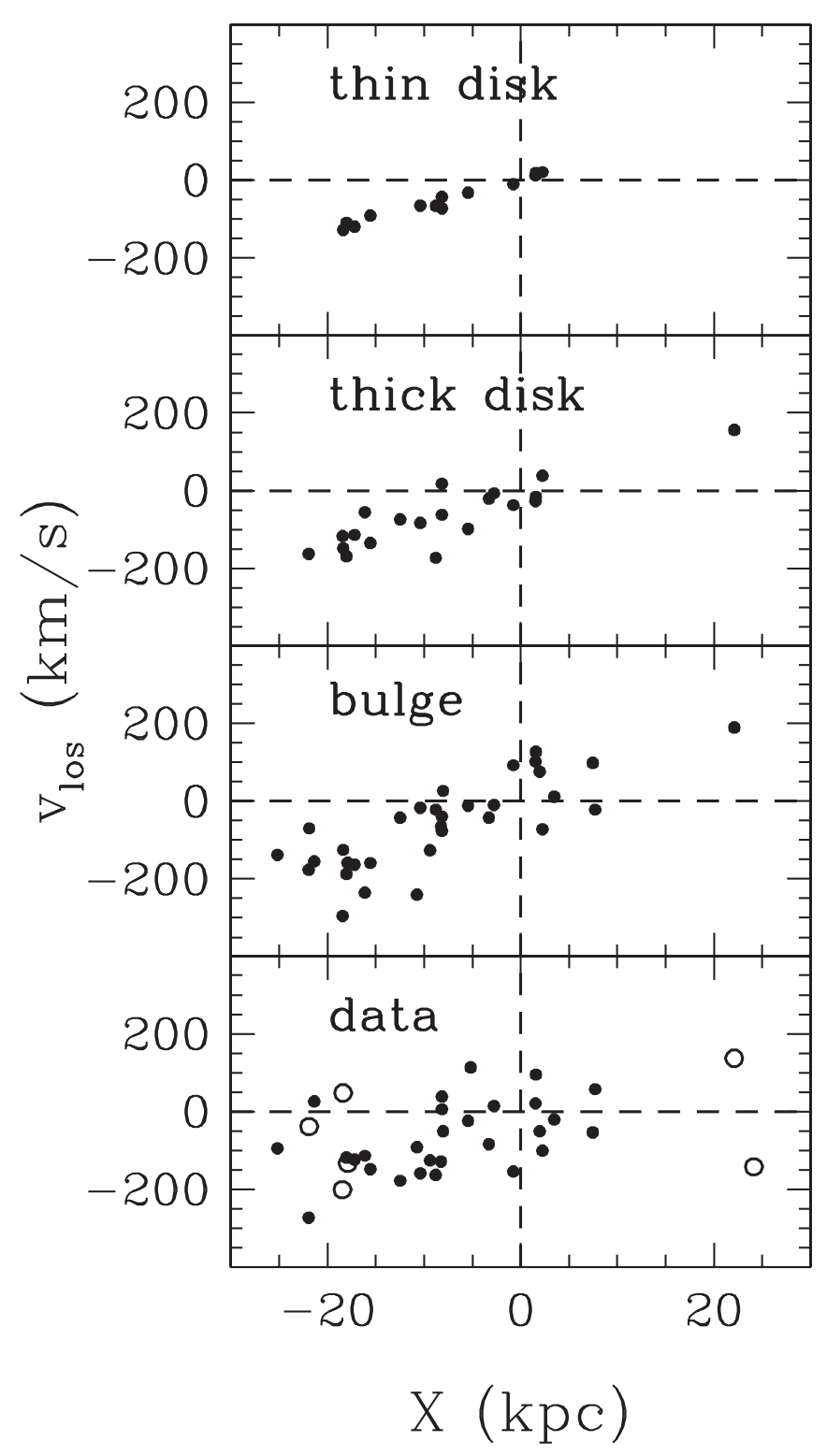

FIG. 9.-Comparison of thin disk, thick disk, and bulge kinematic models and the PN kinematics, for $|Y|>6$. Filled circles are PNs from this survey, and open circles are PNs from NF87. Both the thin and thick disk kinematics have less dispersion at this height above the major axis than the data.

disk since the birth of the globular clusters (Morrison et al. 2003 ); only satellites with $\sim 10 \%$ of the disk mass significantly heat the disk (Quinn \& Goodman 1986; Walker et al. 1996).

The bulge, however, offers a ready explanation. Existing star counts have already hinted that the bulge extends far into the canonical halo. The minor axis profile is well fitted by a single $R^{1 / 4}$ profile as far out as it has been measured $(20 \mathrm{kpc}$; PvdB94). In Figure 10, we have binned the complete subsample of field 1 PNs along the minor axis $(|X|<3 \mathrm{kpc})$ by isophotal radius, assuming that the bulge has an axial ratio of 0.55 . We determined the complete subsample by fitting the known PN luminosity function to the histogrammed data, similar to Ciardullo et al. (1989). At some point, the number of PNs observed falls off from the predicted number of PNs, and this determines our completeness limit. The two PNs from field 2 that are included in this minor axis sample are the two most distant bins in the histogram. The inner three bins are affected
TABLE 5

Milky Way Disk Properties

\begin{tabular}{|c|c|c|c|c|}
\hline Population & $h_{r}$ & $h_{z}$ & $v_{\text {circ }}$ & $\sigma_{r}$ \\
\hline Milky Way thin... & 3.5 & 0.3 & -220 & 30 \\
\hline Milky Way thick ........................ & 4 & 1.0 & -220 & 65 \\
\hline
\end{tabular}

by our exclusion of PNs very near the center of the galaxy, as described earlier.

The minor axis PN distribution is well fitted by the prediction of the PvdB94 surface brightness profile, assuming the M31 PN production rate of Ciardullo et al. (1989) for the upper $\sim 1.5$ mag of the PN luminosity function. It has been suggested that a drop in the PN production rate can be caused by both extreme old age and very low metallicity (Ciardullo 1995; Jacoby et al. 1997; Magrini et al. 2003), as expected in a halo population. We see no dramatic change, however, in the PN production rate along the minor axis out to at least $12 \mathrm{kpc}$. This suggests no sudden decrease in metallicity or age of the dominant population but rather continuity from the central bulge out to at least $12 \mathrm{kpc}$.

With this in mind, we feel justified in considering whether the outer PN velocities are consistent with bulge kinematics. Like the bulges of other early-type spirals, which have $v / \sigma_{v} \sim 1$ (Kormendy \& Illingworth 1982; Davies \& Illingworth 1983), M31 has a moderate degree of rotational support in the inner regions. The central bulge has a large velocity dispersion $(100 \mathrm{~km}$ $\mathrm{s}^{-1}$ ) and a rotation curve that rises to $80 \mathrm{~km} \mathrm{~s}^{-1}$ by about $1 \mathrm{kpc}$ (Richstone \& Shectman 1980). This is significantly less rotation than seen in the data $\left(116 \mathrm{~km} \mathrm{~s}^{-1}\right)$, and a kinematic model based on the central bulge characteristics proves incompatible with our data (Fig. 11).

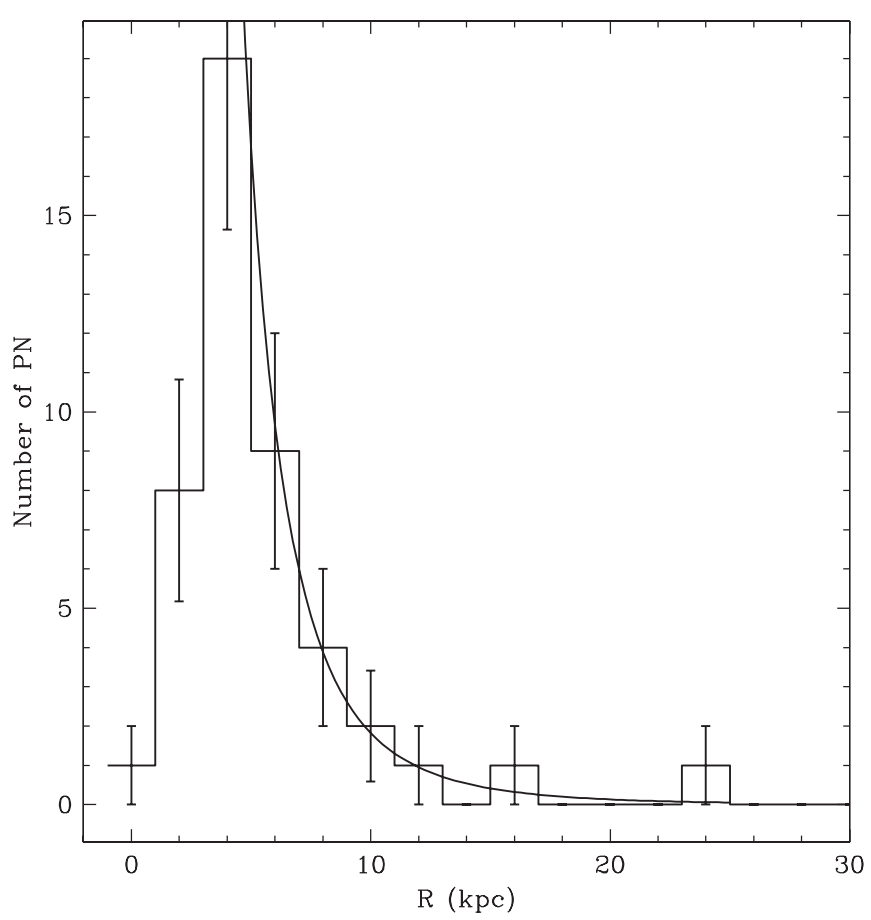

FIG. 10.-Distribution of PNs along the minor axis. PNs in the complete subsample within $3 \mathrm{kpc}$ of the minor axis are assigned an isophotal distance and binned into $2 \mathrm{kpc}$ bins. The solid line is the number of PNs expected based on the minor axis surface brightness profile from PvdB94, normalized to the depth of our survey. The first three bins are reduced by our exclusion of PNs near the center of the galaxy. 

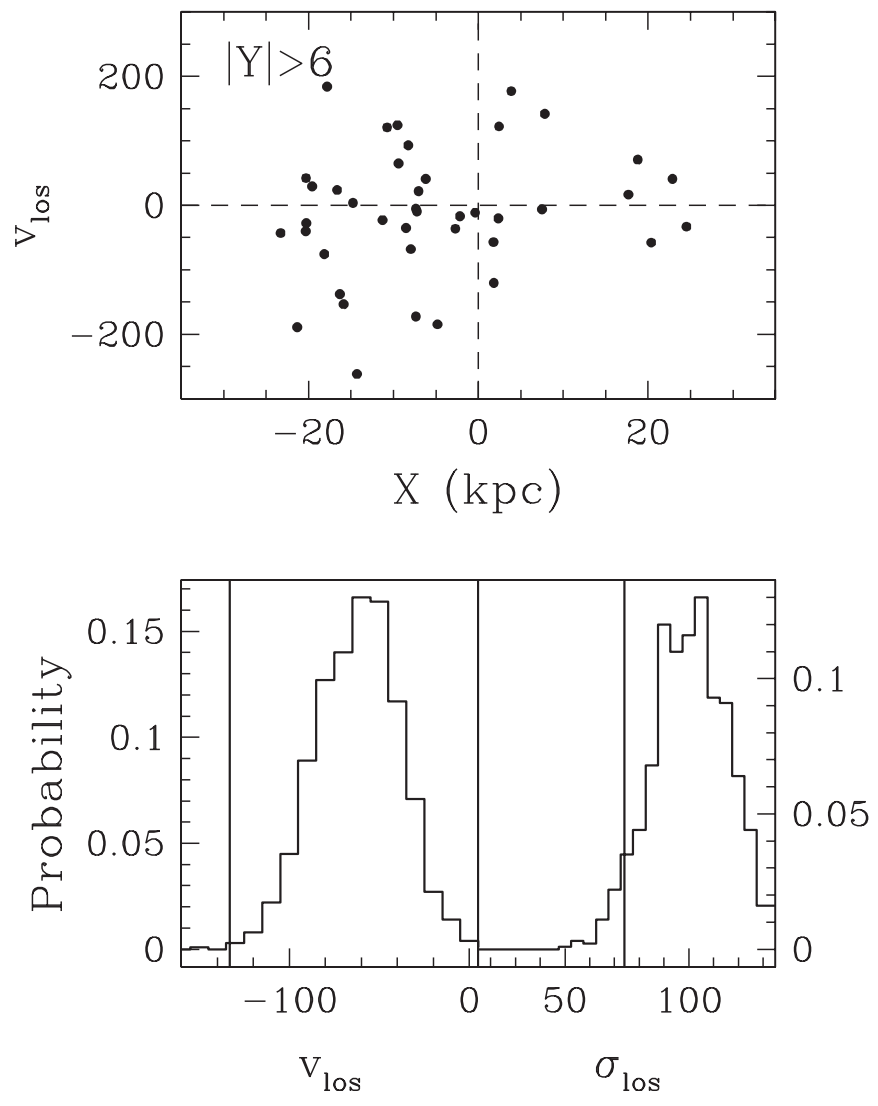

FIG. 11.-Top: One realization of a bulge model with $v_{\text {rot }}=80 \mathrm{~km} \mathrm{~s}^{-1}$ and $\sigma_{r}=100 \mathrm{~km} \mathrm{~s}^{-1}$. Bottom: Probability distribution for the mean $v_{\text {los }}$ and for $\sigma_{\text {los }}$ measured from the positions at $|Y|>6$ and $|X|>10$ for 1000 realizations of the same bulge model as in the top panel. The solid lines represent the values measured from the data, $v_{\text {los }}=116 \mathrm{~km} \mathrm{~s}^{-1}$ and $\sigma_{\mathrm{los}}=79 \mathrm{~km} \mathrm{~s}^{-1}$. All velocities are in kilometers per second.

However, this is not a fair comparison. The properties of the central bulge are based on the nuclear observations of Richstone \& Shectman (1980) and the stellar absorption data of McElroy (1983), which only extend to about $2 \mathrm{kpc}$ along the major axis, less than $1 R_{e}$ for the M31 bulge. These data are on the rising portion of McElroy's rotation curve and in fact show an increase in mean velocity with distance from the center in his Figure 7. Therefore, the bulge rotation could reasonably flatten out at $150-200 \mathrm{~km} \mathrm{~s}^{-1}$ beyond $6 \mathrm{kpc}$ on this side of the galaxy.

Recent observations of low-luminosity elliptical galaxies (Rix et al. 1999) show a similar increase in the degree of rotational support at large radii $\left(>1 R_{e}\right)$, with $v / \sigma=2-3$ in many cases. M31's bulge occupies the same region of the fundamental plane as low-luminosity elliptical galaxies (Kormendy 1985, 1987; Kormendy \& Bender 1999), and we might therefore expect that they have common kinematic properties.

The low-luminosity elliptical data are derived from stellar absorption spectra along the major axis, while our outer PNs are far off-axis. In order to make a better comparison, we have used our kinematic models to predict the value of $v_{\text {rot }}$ and $\sigma_{r}$ that we would expect to observe along the major axis if our PNs belong to a bulge with increasing rotational support. We generated 1000 realizations for each of a set of models covering a range of possible $v_{\text {rot }}$ and $\sigma_{r}$ values and compared the resulting probability distributions for our variables with our observed values.

We found from our models that the line-of-sight dispersion is independent of the rotation velocity in the range of interest and that $\sigma_{r}=50-90 \mathrm{~km} \mathrm{~s}^{-1}$ bracketed the range of reasonable

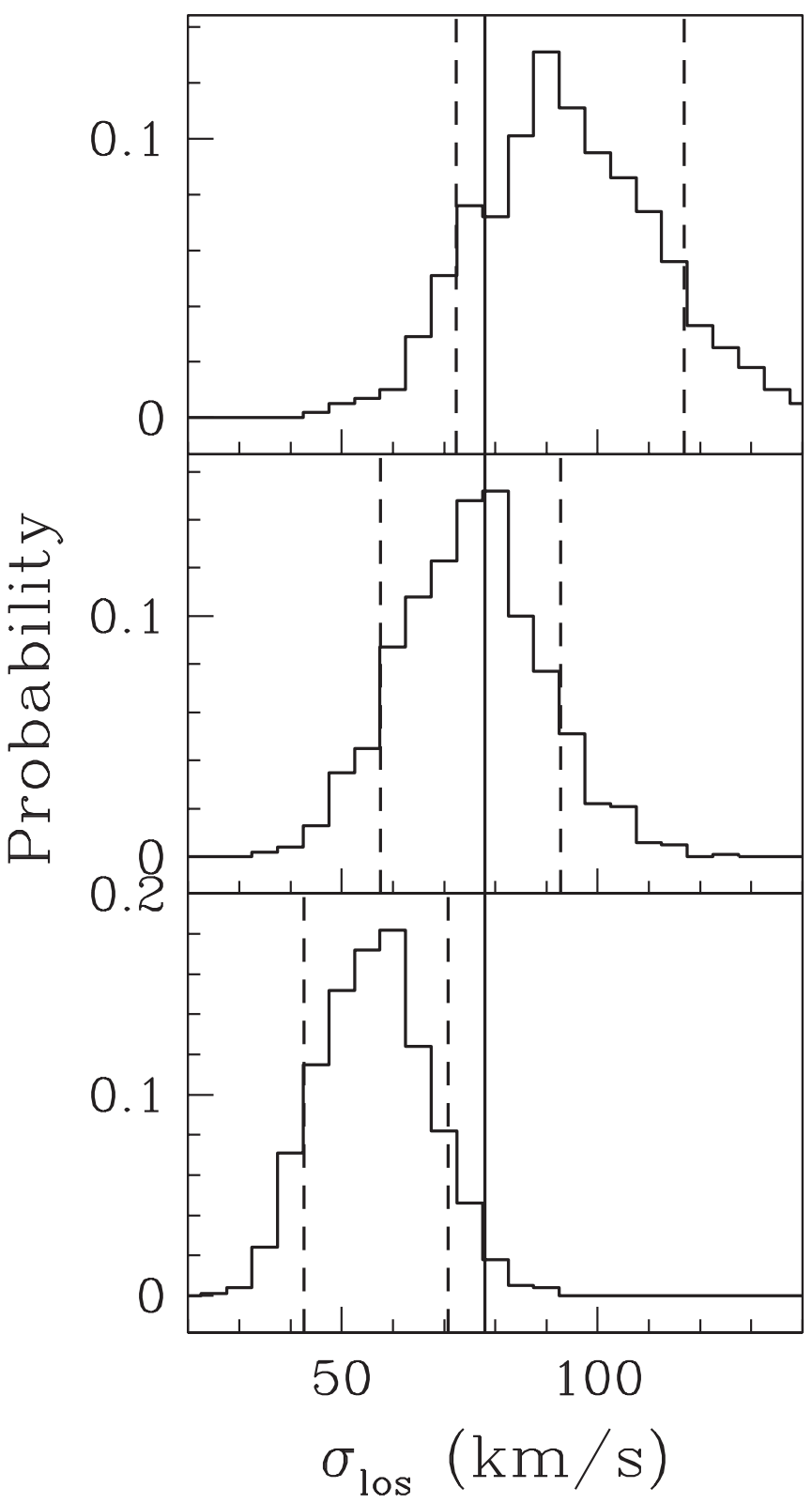

FIG. 12.-Probability distribution for $\sigma_{\text {los }}$ measured from the positions at $|Y|>6$ and $|X|>10$ for 1000 realizations of various bulge models. The solid lines represent the value from the data, $\sigma_{\text {los }}=79 \mathrm{~km} \mathrm{~s}^{-1}$, and the dashed lines are the $10 \%$ confidence limits from the models. A model bulge with $v_{\text {rot }}=160 \mathrm{~km} \mathrm{~s}^{-1}$ and (top) $\sigma_{r}=90 \mathrm{~km} \mathrm{~s}^{-1}$, (middle) $70 \mathrm{~km} \mathrm{~s}^{-1}$, and (bottom) $50 \mathrm{~km} \mathrm{~s}^{-1}$ is shown.

values (Fig. 12). For those values, $v_{\text {rot }}=130-170 \mathrm{~km} \mathrm{~s}^{-1}$ matched $v_{\text {los }}$ (Fig. 13). Our final best estimate is $v_{\text {rot }}=150 \pm$ $20 \mathrm{~km} \mathrm{~s}^{-1}$ and $\sigma_{r}=70 \pm 20 \mathrm{~km} \mathrm{~s}^{-1}$, where the uncertainties cover the range of model parameters that led to a match with the measured values of $v_{\text {los }}$ and $\sigma_{\text {los. }}$. In the lower right panel of Figure 6, we have plotted the range of velocities generated for each PN position in the 1000 model runs of this best model. Only one outer PN is never fitted by the bulge model, in contrast to the disk models. The dark line is the average rotation curve for all the PNs with $|Y|>6$, and the lighter lines are the model $10 \%$ confidence limits for these PNs. Most fall within the limits - their velocities are consistent with our bulge model.

Interestingly, Reitzel et al. (2004) find evidence of a population with similar rotation in a field $34 \mathrm{kpc}$ along the major axis. Roughly two-thirds of their sample of 23 M31 red giants 


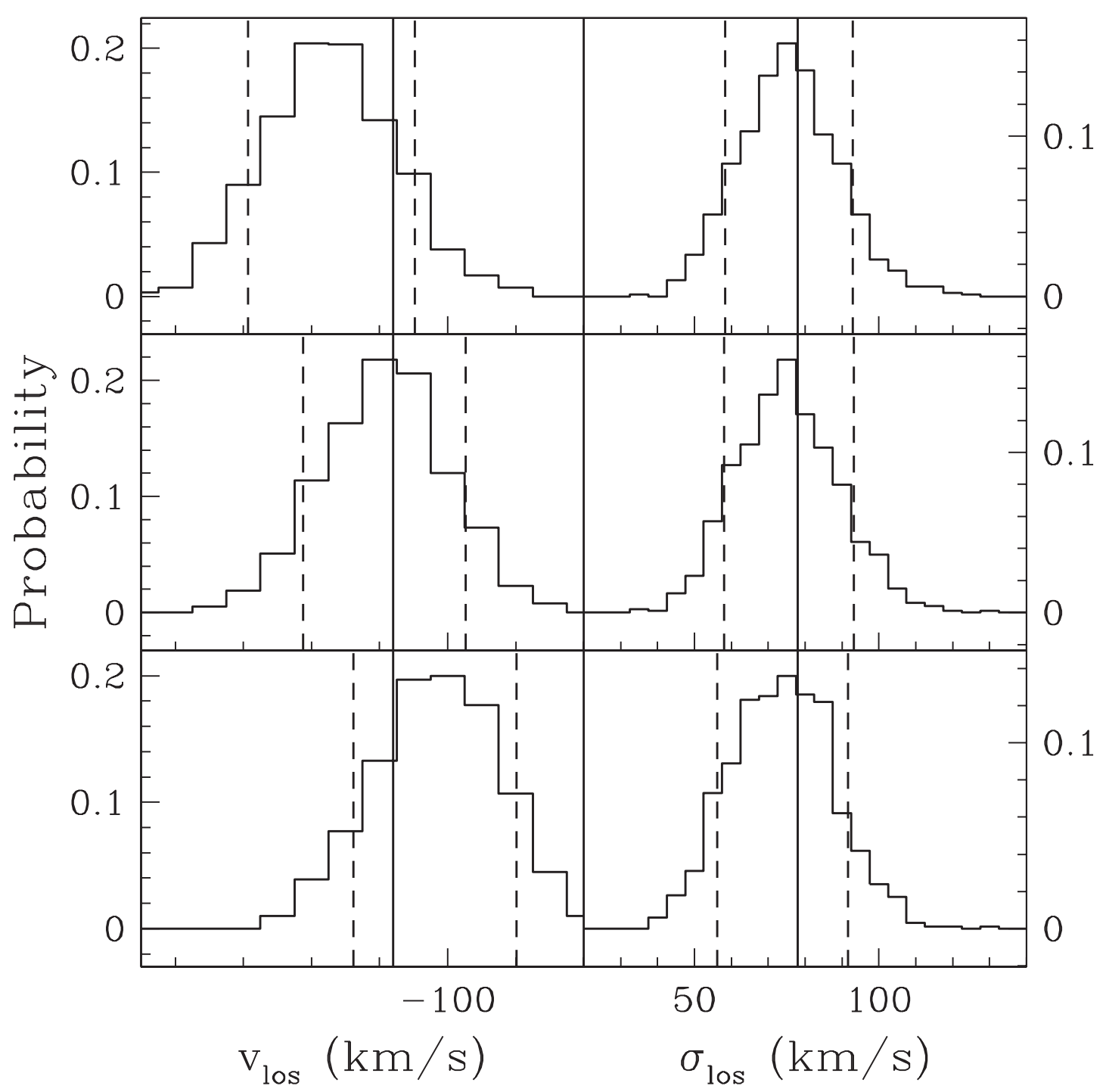

Fig. 13.-Probability distribution for the mean $v_{\text {los }}$ and for $\sigma_{\text {los }}$ measured from the positions at $|Y|>6$ and $|X|>10$ for 1000 realizations of various bulge models. The solid lines represent the values from the data, $v_{\text {los }}=116 \mathrm{~km} \mathrm{~s}^{-1}$ and $\sigma_{\text {los }}=79 \mathrm{~km} \mathrm{~s}^{-1}$, and the dashed lines are the $10 \%$ confidence limits from the models. Top: Model bulge with $v_{\text {rot }}=170 \mathrm{~km} \mathrm{~s}^{-1}$ and $\sigma_{\text {los }}=70 \mathrm{~km} \mathrm{~s}^{-1}$. Middle: Model bulge with $v_{\text {rot }}=150 \mathrm{~km} \mathrm{~s}^{-1}$ and $\sigma_{r}=70 \mathrm{~km} \mathrm{~s}$. Bottom: Model bulge with $v_{\text {rot }}=130 \mathrm{~km} \mathrm{~s}^{-1}$ and $\sigma_{r}=70 \mathrm{~km} \mathrm{~s}^{-1}$.

have velocities relative to M31 of $150 \mathrm{~km} \mathrm{~s}^{-1}$, in good agreement with our model-based estimate for the major axis rotation for the outer bulge. However, the dispersion is low, only $27 \mathrm{~km}$ $\mathrm{s}^{-1}$, and Reitzel et al. (2004) reason that these giants may belong to the disk or to cold substructure in the halo. More velocities covering a wider area will be needed to disentangle these populations.

For the outer PNs, the model suggests $v / \sigma=150 / 70=$ $2.1{ }^{7}$ well within the range covered by the low-luminosity elliptical galaxies (Rix et al. 1999). If we accept that these PNs belong to the M31 bulge on the basis of the surface brightness profile, then the velocities imply that M31's bulge shares kinematic as well as photometric properties with low-luminosity elliptical galaxies. This strengthens the evolutionary connection between these types of galaxies and may provide an important clue to understanding the formation of large spirals like M31.

\footnotetext{
${ }^{7}$ We use $v / \sigma$ in the same sense as Rix et al. (1999): a simple quotient of the line-of-sight velocity and velocity dispersion at a given position.
}

\subsection{The Halo}

Regardless of how we interpret the large rotation velocity of the outer PNs, it seems clear that most do not belong to a kinematically hot, canonical halo. Are there any PNs in our sample that could? Without recourse to more detailed models than are justified by the data, the best we can do is identify PNs that cannot belong to either the disk or the bulge. At all $Y$, a few objects can be found that have velocities that are inconsistent with either of these populations, and their coordinates and velocities are listed in Table 6 . As a group, their spectra do not systematically differ from the other PNs.

The surprising conclusion we draw from the PN velocities and Figure 14, where we have overplotted their positions on Figure 2 from Ferguson et al. (2002), is that all five halo PNs probably belong to tidal streams in M31's outer halo. One is projected on the Northern Spur and is counterrotating with respect to the disk there. Two are projected near the major axis at $X=-10 \mathrm{kpc}$; their velocities are similar to M32's systemic velocity, and they could be debris from that galaxy. All three of these halo PNs have velocities in the "forbidden" quadrants. 
TABLE 6

Coordinates and Heliocentric Velocities for Halo PN Candidates IN M31

\begin{tabular}{ccccr}
\hline \hline ID & R.A. (2000.0) & Decl. (2000.0) & $v_{\text {helio }}$ & $v_{\text {err }}$ \\
\hline HKPN 86 .... & 004256.02 & 405112.5 & -638.3 & 5.3 \\
HKPN 85 ..... & 004255.60 & 410340.9 & -599.1 & 5.6 \\
HKPN 32 .... & 003949.08 & 404239.5 & -168.8 & 28.6 \\
HKPN 41 .... & 004014.24 & 402323.9 & -126.1 & 2.5 \\
NF .............. & 004626.51 & 430042.15 & -452.3 & $\ldots$ \\
\hline
\end{tabular}

Notes.- The last object is from the study of Nolthenius \& Ford (1987). Units of right ascension are hours, minutes, and seconds, and units of declination are degrees, arcminutes, and arcseconds.

The remaining two halo PNs are located near the center of the galaxy. Their velocities are within $40 \mathrm{~km} \mathrm{~s}^{-1}$ of each other $\left(v_{\text {helio }} \sim-600 \mathrm{~km} \mathrm{~s}^{-1}\right)$ and are at least 3 times the central bulge dispersion $\left(100 \mathrm{~km} \mathrm{~s}^{-1}\right)$. Orbits suggested by Merrett et al. (2003) and Ibata et al. (2004) for the Southern Stream (Ibata et al. 2001; Ferguson et al. 2002) project near the position of these PNs. Their velocities follow the gradient found by Ibata et al. (2004), implying that these PNs could belong to the Southern Stream. Finding PNs in low surface brightness streams is somewhat unexpected, since PNs are intrinsically rare objects (only $\sim 30 / 10^{9} L_{\odot}$; Ciardullo et al. 1989). Velocities in the streams will provide constraints on the orbits of their progenitors, and deeper observations are planned to detect more stream PNs. We will revisit these possible streams in a later paper.

We have yet to find the M31 analog to the hot component of the Milky Way inner halo. If the PN production rate per unit luminosity is the same as for other M31 populations, we would expect to see on the order of 20-30 PNs if we covered the entire halo of M31 at the depth of our survey, and its luminosity is on the order of $10^{9} L_{\odot}$, the approximate luminosity of the Milky Way halo (Morrison \& Harding 1993). Depending on the density distribution of the halo stars, we have surveyed onequarter or less of the halo, so very roughly 5-7 PNs would be expected in our sample from such a halo. Yet all five of our halo PN candidates belong to kinematically cold features in the halo.

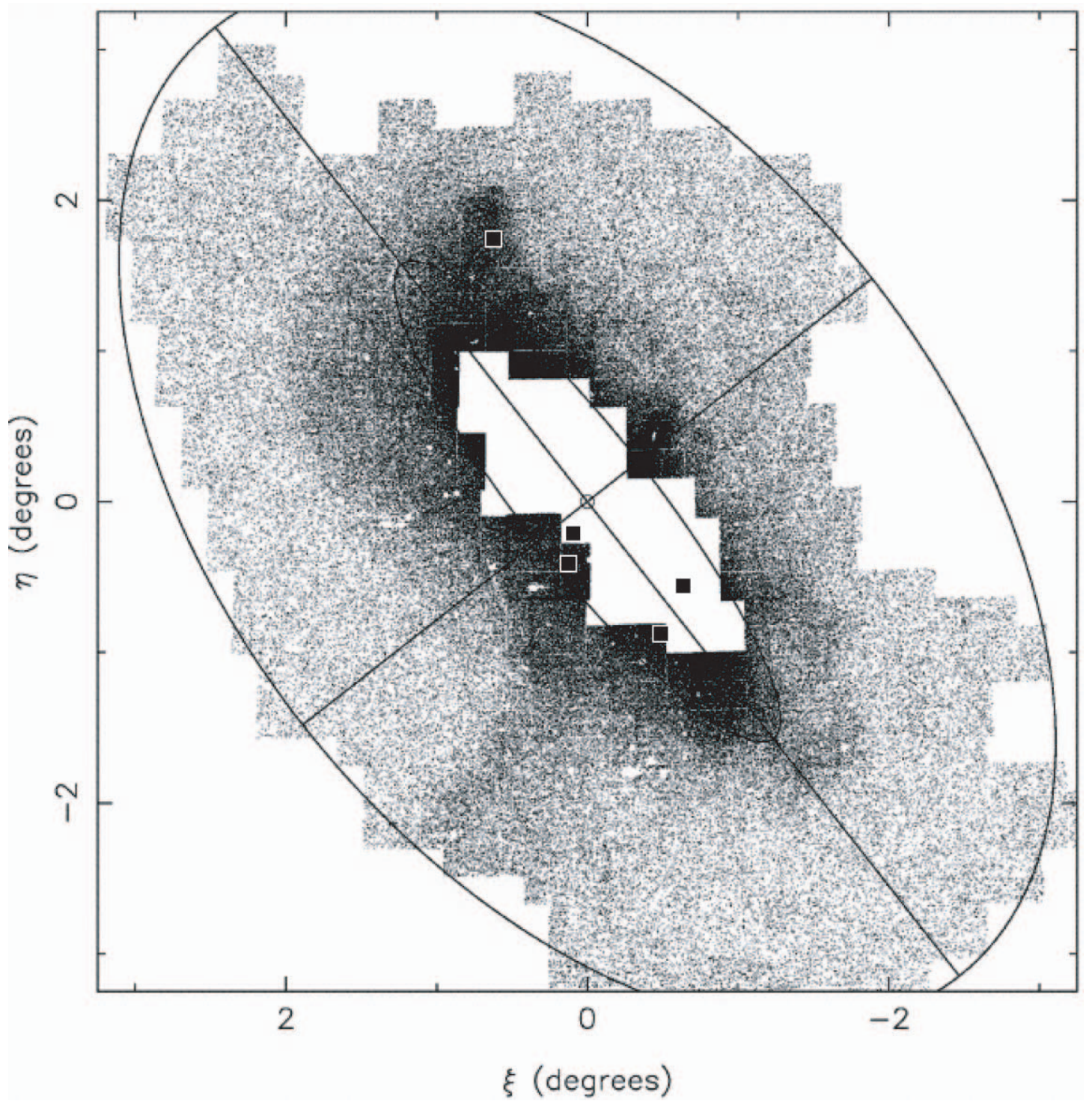

FIG. 14.-Fig. 2 from Ferguson et al. (2002), showing M31's halo and tidal streams, with the positions of our potential halo objects marked. All five fall on or near possible tidal streams, and three are counterrotating with respect to the disk at their position. The two halo candidates near the center have velocities within $40 \mathrm{~km} \mathrm{~s}^{-1}$

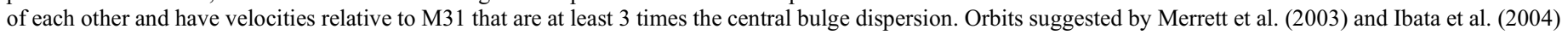

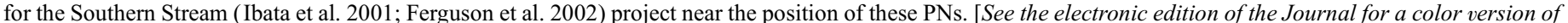
this figure.] 
On the surface, this suggests that the M31 halo does not have a kinematically hot component analogous to the inner Milky Way halo. However, if the hot component of the M31 halo is as old and metal-poor as the Milky Way halo, the PN production rate could be lower by a factor of 5 or more (Magrini et al. 2003). This would be consistent with a total lack of hot halo objects in our sample. Studies in search of these objects must reach farther down the PN luminosity function by several magnitudes.

Spectroscopic surveys of red giant stars, because of the larger sample size, may be needed to find the hot halo, especially if the PN production rate is significantly lower for old populations. Reitzel \& Guhathakurta (2002) found a high dispersion of $150 \mathrm{~km} \mathrm{~s}^{-1}$ in a sample of M31 red giants in a field $19 \mathrm{kpc}$ along the minor axis. This field is farther out than our PN sample and may have reached, at last, a region where the halo dominates.

\section{DISCUSSION}

\subsection{Bulge versus Halo}

The extent of M31's bulge has been a topic of debate in the literature. Some previous studies of M31 stellar populations suggested that fields as far out as $20 \mathrm{kpc}$ were still dominated by the bulge (Mould \& Kristian 1986; Durrell et al. 2001; Freeman 1996), primarily because of two important findings: first, the higher metallicity of the stars compared to the Milky Way halo at comparable radius (Durrell et al. 1994, 2001; Holland et al. 1996), and second, its $R^{1 / 4}$ surface brightness profile, which extends smoothly out to at least $20 \mathrm{kpc}$ on the minor axis (PvdB94). We add to this evidence the discovery that PNs at $|Y|>6 \mathrm{kpc}$, in regions typically associated with the halo, have a $v_{\text {los }}=116 \mathrm{~km} \mathrm{~s}^{-1}$ and $\sigma_{\text {los }}=79 \mathrm{~km} \mathrm{~s}^{-1}$. This rotation is very different from the behavior of the Milky Way halo populations at similar radii and suggests that the bulge light dominates even out to $20 \mathrm{kpc}$ on the minor axis.

M31 globular clusters also differ significantly from their Milky Way counterparts. As a system, the clusters have a large rotation, $\sim 150 \mathrm{~km} \mathrm{~s}^{-1}$ on average (Perrett et al. 2002). This holds for both the metal-rich and the metal-poor subsets, implying that neither have clear "halo" kinematics, although the precise velocity data set of Perrett et al. (2002) was confined to inner regions and may select against halo objects. Morrison et al. (2004) demonstrate that $\sim 50 \%$ of M31 clusters projected on the disk actually have thin disk kinematics. No analogous population is known among the Milky Way globular clusters. In contrast, studies of the Milky Way globular clusters have found that metal-poor clusters belong to the halo (Zinn 1985), while the central metal-rich clusters have either bulge or thick disk kinematics (Frenk \& White 1982; Minniti 1995; Côté 1999; Armandroff 1989).

If canonical M31 halo regions are actually dominated by the bulge, then further comparisons with the Milky Way halo are misleading. Instead, these M31 halo regions provide a baseline for comparison with more distant early-type spiral bulges. How does a bulge with moderate rotational support form? In major mergers, angular momentum can migrate outward in the dark halo as it is assembled (Quinn \& Zurek 1988). Thus, a bulge that formed in the very early stages of galaxy formation simultaneously with the dark halo assembly would have the $R^{1 / 4}$ law profile characteristic of violent relaxation, as well as increased rotation at large radii. PN studies of the outer regions of some large elliptical galaxies show this expected increase in rotation with radius (e.g., Hui et al. 1993).
Dissipationless mergers of disk galaxies of unequal mass (ratio 3:1) can also lead to disky remnants with more rotational support in the inner regions and $R^{1 / 4}$ law profiles (Naab et al. 1999; Bendo \& Barnes 2000) since the larger disk partly survives the interaction. We find that the kinematics of M31's outer bulge is in rough agreement with the low-luminosity elliptical observations. A comparison of M31's bulge kinematics with these observations is complicated because velocities for the elliptical galaxies were measured along the major axis. Nonetheless, we find that $v / \sigma=2.1$ by $5 R_{e}$ on the basis of the outer bulge PN kinematics in M31 (assuming $R_{e}=$ $2.4 \mathrm{kpc}$ on the major axis; PvdB94), similar to values for the low-luminosity elliptical galaxies, suggesting an evolutionary kinship.

\subsection{The Elusive Halo Remains Elusive}

In our entire survey, we only find a handful of PNs in the combined sample with halo-like kinematics. Ideally, these are the sample we should compare with the Milky Way halo; however, our PN spectra are not suitable for abundances, and they tell us nothing about ages. In principle, they offer the possibility of comparing the luminosities of the M31 and Milky Way halos because the PNs are direct tracers of the underlying stars. However, this is a difficult comparison to make for several reasons. First, uncertainties in the PN production rate make it difficult to translate the number of PNs to stellar luminosity. Second, hot halo objects can have any velocity, and confusion with the disk and bulge prevent us from unambiguously identifying those not in the forbidden quadrants. Third, the Milky Way halo luminosity is only approximately known, and depends strongly on the density of the little-studied inner halo because the halo density distribution is extremely centrally concentrated, with a globular cluster density proportional to $r^{-3.5}$ (Zinn 1985).

Perhaps most important, a generalization from our survey to the entire halo is not appropriate if M31's halo is clumpy. In fact, we know this to be an important issue, since substructure in field star counts has been detected in M31's halo (Ibata et al. 2001; Ferguson et al. 2002). The clumpiness of the Milky Way halo is now accepted on the basis of the disruption of the Sgr dSph (Ibata et al. 1994; Ivezić et al. 2000; Yanny et al. 2000; Dohm-Palmer et al. 2001) and of studies of velocity substructures among the metal-poor halo giants in the solar neighborhood (Helmi et al. 1999).

The PN observations thus far tell us more about the bulge than the halo, as do other studies of the stellar populations in these fields. The results of such studies can now be associated with the bulge, for example, estimates of a surprisingly high halo luminosity (Reitzel et al. 1998), an intermediate-age component (Brown et al. 2003), and moderate flattening (PvdB94; Ferguson et al. 2000).

\section{SUMMARY}

Our search for M31's halo has led to a picture of that galaxy very different from that of the Milky Way. PNs at large distances from the M31 major axis have significant rotation and small velocity dispersion, in contrast to the large velocity dispersion of the Milky Way halo. We conclude that these objects do not belong to the halo but to the outer reaches of the bulge.

If this is the case, then among other characteristics, M31's bulge shares the rotation properties of low-luminosity elliptical galaxies, namely, that the amount of rotational support increases with radius. This suggests an evolutionary kinship with 
low-luminosity elliptical galaxies, which are believed to have formed via hierarchical merging of objects of nearly equal mass, in contrast to the Milky Way halo, which formed from the on-going accretion of very small satellites.

Any kinematically hot halo in M31 is represented by a very small number of objects in our sample. Yet given the rarity of PNs, even these few could imply a halo luminosity comparable to that of the Milky Way if they belong to a smooth inner component. However, their projection on M31 suggests that they may instead belong to tidal streams in a clumpy halo. We are not claiming that M31 does not have a Milky Way-like halo. Rather, we support, as some earlier researchers have surmised, that the halo is dominated by the bulge out to $20 \mathrm{kpc}$ and farther; preliminary star counts of red giants in a field at $30 \mathrm{kpc}$ reveal a metallicity distribution function similar to that of nearer regions (P. Durrell 2003, private communication).

Sorting out M31's evolutionary history will require more data, and PNs provide the means. With a larger sample of PN velocities, we can clarify the distinction between bulge and halo by connecting the kinematics to the spatial distribution. Most important, abundances can be derived for our sample of halo objects. This is a great advantage of PNs; they can ultimately provide a large number of abundances (e.g., Jacoby \& Ciardullo 1999), where this is costly in telescope time for field stars (Reitzel \& Guhathakurta 2002). The ability to tie abundance to kinematics is a connection that has been critical to our understanding of the stellar populations of the Milky Way.

We thank Rene Walterbos for providing the surface photometry of M31 and the image used in Figure 2, Annette Ferguson for the use of Figure 2 from Ferguson et al. (2002), and John Feldmeier and Chris Mihos for many useful discussions. H. L. M. acknowledges the support of NSF CAREER grant AST 96-24542. D. H. K. is supported by an NSF Astronomy and Astrophysics Postdoctoral Fellowship under award AST 01-04455.
Armandroff, T. E. 1989, AJ, 97, 375

Arnaboldi, M., Freeman, K. C., Gerhard, O., Matthias, M., Kudritzki, R. P., Mendez, R. H., Capaccioli, M., \& Ford, H. 1998, ApJ, 507, 759

Arnaboldi, M., et al. 1996, ApJ, 472, 145

Barmby, P., Huchra, J. P., Brodie, J. P., Forbes, D. A., Schroder, L. L., \& Grillmair, C. J. 2000, AJ, 119, 727

Beers, T. C., Chiba, M., Yoshii, Y., Platais, I., Hanson, R. B., Fuchs, B., \& Rossi, S. 2000, AJ, 119, 2866

Bendo, G. J., \& Barnes, J. E. 2000, MNRAS, 316, 315

Berman, S., \& Loinard, L. 2002, MNRAS, 336, 477

Binney, J., \& Tremaine, S. 1987, Galactic Dynamics (Princeton: Princeton Univ. Press)

Bottema, R. 1993, A\&A, 275, 16

Braun, R. 1991, ApJ, 372, 54

Brinks, E., \& Burton, W. B. 1984, A\&A, 141, 195

Brinks, E., \& Shane, W. W. 1984, A\&AS, 55, 179

Brown, T. M., Ferguson, H. C., Smith, E., Kimble, R. A., Sweigart, A. V., Renzini, A., Rich, R. M., \& VandenBerg, D. A. 2003, ApJ, 592, L17

Ciardullo, R. 1995, in IAU Highlights of Astronomy 10, ed. I. Appenzeller (Dordrecht: Kluwer), 507

Ciardullo, R., Jacoby, G. H., Ford, H. C., \& Neill, J. D. 1989, ApJ, 339, 53

Côté, P. 1999, AJ, 118, 406

Courteau, S., de Jong, R. S., \& Broeils, A. H. 1996, ApJ, 457, L73

Couture, J., Racine, R., Harris, W. E., \& Holland, S. 1995, AJ, 109, 2050

Cram, T. R., Roberts, M. S., \& Whitehurst, R. N. 1980, A\&AS, 40, 215

Davies, R. L., \& Illingworth, G. 1983, ApJ, 266, 516

de Vaucouleurs, G. 1958, ApJ, 128, 465

de Vaucouleurs, G., de Vaucouleurs, A., Corwin, H. G., Jr., Buta, R. J.,

Paturel, G., \& Fouqu, P. 1991, Third Reference Catalogue of Bright Galaxies

(New York: Springer)

Dohm-Palmer, R. C., et al. 2001, ApJ, 555, L37

Drimmel, R., \& Spergel, D. N. 2001, ApJ, 556, 181

Durrell, P. R., Harris, W. E., \& Pritchet, C. J. 1994, AJ, 108, 2114 2001, AJ, 121,2557

2004, AJ, 128, 260

Edvardsson, B., Andersen, J., Gustafsson, B., Lambert, D. L., Nissen, P. E., \& Tomkin, J. 1993, A\&A, 275, 101

Eggen, O. J., Lynden-Bell, D., \& Sandage, A. R. 1962, ApJ, 136, 748

Ferguson, A. M. N., Gallagher, J. S., \& Wyse, R. F. G. 2000, AJ, 120, 821

Ferguson, A. M. N., Irwin, M. J., Ibata, R. A., Lewis, G. F., \& Tanvir, N. R. 2002, AJ, 124, 1452

Ford, H. C., Jacoby, G., \& Jenner, D. C. 1977, ApJ, 213, 18

Freedman, W. L., \& Madore, B. F. 1990, ApJ, 365, 186

Freeman, K. C. 1996, in ASP Conf. Ser. 92, Formation of the Galactic Halo . . Inside and Out, ed. G. W. Preston, H. Morrison, \& A. Sarajedini (San Francisco: ASP), 3

Frenk, C. S., \& White, S. D. M. 1982, MNRAS, 198, 173

Gilmore, G., \& Reid, N. 1983, MNRAS, 202, 1025

Helmi, A., White, S. D. M., de Zeeuw, P. T., \& Zhao, H. 1999, Nature, 402, 53

Holland, S., Fahlman, G. G., \& Richer, H. B. 1996, AJ, 112, 1035

\section{EFERENCES}

Huchra, J. P. 1993, in ASP Conf. Ser. 48, The Globular Cluster-Galaxy Connection, ed. G. H. Smith \& J. P. Brodie (San Francisco: ASP), 420

Huchra, J. P., Brodie, J. P., \& Kent, S. M. 1991, ApJ, 370, 495

Huchra, J. P., Vogeley, M. S., \& Geller, M. J. 1999, ApJS, 121, 287

Hui, X., Ford, H. C., Ciardullo, R., \& Jacoby, G. H. 1993, ApJ, 414, 463

Hui, X., Ford, H. C., Freeman, K. C., \& Dopita, M. A. 1995, ApJ, 449, 592

Hui, X., Ford, H., \& Jacoby, G. 1994, BAAS, 26, 938

Ibata, R., Chapman, S., Ferguson, A. M. N., Irwin, M., Lewis, G., \& McConnachie, A. 2004, MNRAS, 351, 117

Ibata, R., Irwin, M., Lewis, G., Ferguson, A. M. N., \& Tanvir, N. 2001, Nature, 412,49

Ibata, R. A., Gilmore, G., \& Irwin, M. J. 1994, Nature, 370, 194

Ivezić, Z., et al. 2000, AJ, 120, 963

Jacoby, G. H., \& Ciardullo, R. 1999, ApJ, 515, 169

Jacoby, G. H., Ciardullo, R., Booth, J., \& Ford, H. C. 1989, ApJ, 344, 704

Jacoby, G. H., Morse, J. A., Fullton, L. K., Kwitter, K. B., \& Henry, R. B. C. 1997, AJ, 114, 2611

Jacoby, G. H., et al. 1992, PASP, 104, 599

Jarrett, T. H., Chester, T., Cutri, R., Schneider, S. E., \& Huchra, J. P. 2003, AJ, 125,525

Kent, S. M. 1989, AJ, 97, 1614

Kormendy, J. 1985, ApJ, 295, 73

- 1987, in Nearly Normal Galaxies: From the Planck Time to the Present, ed. S. M. Faber (New York: Springer), 163

Kormendy, J., \& Bender, R. 1999, ApJ, 522, 772

Kormendy, J., \& Illingworth, G. 1982, ApJ, 256, 460

Kulkarni, S. R., Heiles, C., \& Blitz, L. 1982, ApJ, 259, L63

Laird, J. B., Carney, B. W., Rupen, M. P., \& Latham, D. W. 1988, AJ, 96, 1908

Loinard, L., Dame, T. M., Heyer, M. H., Lequeux, J., \& Thaddeus, P. 1999, A\&A, 351, 1087

Lopez-Corredoira, M., Cabrera-Lavers, A., Garzon, F., \& Hammersly, P. L. 2002, A\&A, 394, 883

Magrini, L., et al. 2003, A\&A, 407, 51

Majewski, S. R., Munn, J. A., \& Hawley, S. L. 1994, ApJ, 427, L37

Mateo, M. L. 1998, ARA\&A, 36, 435

McElroy, D. B. 1983, ApJ, 270, 485

Méndez, R. H., Riffeser, A., Kudritzki, R.-P., Matthias, M., Freeman, K. C., Arnaboldi, M., Capaccioli, M., \& Gerhard, O. E. 2001, ApJ, 563, 135

Merrett, H. R., et al. 2003, MNRAS, 346, L62

Minniti, D. 1995, AJ, 109, 1663

Monet, D., et al. 1998, The USNO-A2.0 Catalogue (Washington: USNO)

Morrison, H. L. 1999, in ASP Conf. Ser. 165, Third Stromlo Symp., The Galactic Halo, ed. B. K. Gibson, T. S. Axelrod, \& M. E. Putman (San Francisco: ASP), 174

Morrison, H. L., Boroson, T. A., \& Harding, P. 1994, AJ, 108, 1191

Morrison, H. L., \& Harding, P. 1993, PASP, 105, 977

Morrison, H. L., Harding, P., Hurley-Keller, D. \& Jacoby, G., 2003, ApJ, 596, L183

Morrison, H. L., Harding, P., Perrett, K., \& Hurley-Keller, D. 2004, ApJ, 603, 87

Mould, J. 1986, in Stellar Populations, ed. C. A. Norman \& A. Renzini (Cambridge: Cambridge Univ. Press), 9 
Mould, J., \& Kristian, J. 1986, ApJ, 305, 591

Naab, T., Burkert, A., \& Hernquist, L. 1999, ApJ, 523, L133

Neeser, M. J., Sackett, P. D., De Marchi, G., \& Paresce, F. 2002, A\&A, 383,472

Nolthenius, R., \& Ford, H. 1986, ApJ, 305, 600

Nolthenius, R., \& Ford, H. C. 1987, ApJ, 317, 62 (NF87)

Norris, J. 1986, ApJS, 61, 667

1999, Ap\&SS, 265, 213

Oey, M. S. 2000, ApJ, 542, L25

Olling, R. 1996, AJ, 112, 457

Pellet, A., Astier, N., Viale, A., Courtes, G., Maucherat, A., Monnet, G., \& Simien, F. 1978, A\&AS, 31, 439

Perrett, K. M., Bridges, T. J., Hanes, D. A., Irwin, M. J., Brodie, J. P., Carter, D., Huchra, J. P., \& Watson, F. G. 2002, AJ, 123, 2490

Pfenniger, D., \& Norman, C. 1990, ApJ, 363, 391

Pohlen, M., Balcells, M., Lütticke, R., \& Dettmar, R. J. 2004, A\&A, 422, 465

Pritchet, C. J., \& van den Bergh, S. 1994, AJ, 107, 1730 (PvdB94)

Quinn, P. J., \& Goodman, J. 1986, ApJ, 309, 472

Quinn, P. J., \& Zurek, W. H. 1988, ApJ, 331, 1

Reitzel, D. B., \& Guhathakurta, P. 2002, AJ, 124, 234

Reitzel, D. B., Guhathakurta, P., \& Gould, A. 1998, AJ, 116, 707
Reitzel, D. B., Guhathakurta, P., \& Rich, R. M. 2004, AJ, 127, 2133

Rich, R. M., Mighell, K. J., Freedman, W. L., \& Neill, J. D. 1996, AJ, 111, 768

Richstone, D. O., \& Shectman, S. A. 1980, ApJ, 235, 30

Rix, H., Carollo, C. M., \& Freeman, K. 1999, ApJ, 513, L25

Roberts, M. S., \& Whitehurst, R. N. 1975, ApJ, 201, 327

Ryan, S. G., \& Norris, J. E. 1991, AJ, 101, 1865

Saha, A. 1985, ApJ, 289, 310

Sancisi, R., \& Allen, R. J. 1979, A\&A, 74, 73

Schechter, P. L., Mateo, M., \& Saha, A. 1993, PASP, 105, 1342

Searle, L., \& Zinn, R. 1978, ApJ, 225, 357

Stark, A. A., \& Binney, J. 1994, ApJ, 426, L31

van der Kruit, P. C., \& Freeman, K. C. 1984, ApJ, 278, 81

van der Kruit, P. C., \& Searle, L. 1981, A\&A, 95, 105

Walker, I. R., Mihos, J. C., \& Hernquist, L. 1996, ApJ, 460, 121

Walterbos, R. A. M., \& Kennicutt, R. C., Jr. 1988, A\&A, 198, 61 (WK88)

Weiland, J. L., et al. 1994, ApJ, 425, L81

Yanny, B., et al. 2000, ApJ, 540, 825

Zepf, S. E., Liu, M. C., Marleau, F. R., Sackett, P. D., \& Graham, J. R. 2000, AJ, 119, 1701

Zinn, R. 1985, ApJ, 293, 424

Zucker, D., et al. 2004, ApJ, 612, L117 